\title{
An examination of two pathways to tropical cyclogenesis occurring in idealized simulations with a cloud-resolving numerical model
}

\author{
M. E. Nicholls ${ }^{1}$ and M. T. Montgomery ${ }^{2,3}$ \\ ${ }^{1}$ University of Colorado, Co-operative Institute for Research in Environmental Sciences, Boulder, Colorado, USA \\ ${ }^{2}$ Department of Meteorology, Naval Postgraduate School, Monterey, California, USA \\ ${ }^{3}$ NOAA's Hurricane Research Division, Miami, Florida, USA
}

Correspondence to: M. E. Nicholls (melville.nicholls@ colorado.edu)

Received: 31 May 2012 - Published in Atmos. Chem. Phys. Discuss.: 9 January 2013

Revised: 3 April 2013 - Accepted: 22 May 2013 - Published: 21 June 2013

\begin{abstract}
Simulations are conducted with a cloud-resolving numerical model to examine the transformation of a weak incipient mid-level cyclonic vortex into a tropical cyclone. Results demonstrate that two distinct pathways are possible and that development along a particular pathway is sensitive to model physics and initial conditions. One pathway involves a steady increase of the surface winds to tropical cyclone strength as the radius of maximum winds gradually decreases. A notable feature of this evolution is the creation of small-scale lower tropospheric cyclonic vorticity anomalies by deep convective towers and subsequent merger and convergence by the low-level secondary circulation. The second pathway also begins with a strengthening low-level circulation, but eventually a significantly stronger mid-level circulation develops. Cyclogenesis occurs subsequently when a small-scale surface concentrated vortex forms abruptly near the center of the larger-scale circulation. The small-scale vortex is warm core throughout the troposphere and results in a fall in local surface pressure of a few millibars. It usually develops rapidly, undergoing a modest growth to form a small tropical cyclone. Many of the simulated systems approach or reach tropical cyclone strength prior to development of a prominent mid-level vortex so that the subsequent formation of a strong small-scale surface concentrated vortex in these cases could be considered intensification rather than genesis.

Experiments are performed to investigate the dependence on the inclusion of the ice phase, radiation, the size and strength of the incipient mid-level vortex, the amount of moisture present in the initial vortex, and the sea surface temperature. Notably, as the sea surface temperature is raised, the likelihood of development along the second pathway is in-
\end{abstract}

creased. This appears to be related to an increased production of ice. The sensitivity of the pathway taken to model physics and initial conditions revealed by these experiments raise the possibility that the solution to this initial value problem is near a bifurcation point. Future improvements to model parameterizations and more accurate observations of the transformation of disturbances to tropical cyclones should clarify the conditions that favor a particular pathway when starting from a mid-level vortex.

\section{Introduction}

The problem of how a tropical disturbance transforms into a tropical cyclone (TC) has been an active field of investigation for over fifty years. Numerical modeling has become an increasingly useful research tool, as more powerful computer processing and the development of advanced cloud models enable more accurate simulation of microphysical processes, air-sea interaction and radiative exchange. Relatively recent studies that have attempted to resolve clouds, rather than parameterize their effects, show significant ability to reproduce observed characteristics of mature TCs (Liu et al., 1997; Braun, 2002; Rogers et al., 2003).

Hendricks et al. (2004, hereafter H04) extended the genesis study of Davis and Bosart (2001) by analyzing a high-resolution ( $3 \mathrm{~km}$ horizontal grid spacing) nearcloud-resolving numerical simulation of Hurricane Diana (1984) with the fifth generation Pennsylvania State University-National Center for Atmospheric Research (PSUNCAR) Mesoscale Model (MM5). The numerical model was 
initialized with an analysis of observed data at a stage when the system that would evolve into Hurricane Diana was a subtropical disturbance. Results suggested that convective plumes with intense vertical vorticity in their cores were the preferred convective structures. The term vortical hot tower (VHT) was coined to describe these structures. H04 suggested that VHTs play an important role in a two-stage evolutionary process: (1) preconditioning of the local environment via diabatic production of multiple small-scale lower tropospheric cyclonic potential vorticity (PV) anomalies, and (2) multiple mergers and axisymmeterization of these low-level $\mathrm{PV}$ anomalies. In addition to the organizational process of the $\mathrm{PV}$ anomalies, the cyclogenesis is enhanced by the aggregate diabatic heating associated with the VHTs, which produce a net influx of low-level mean angular momentum throughout the genesis.

Similar results to H04 were obtained in a study by Montgomery et al. (2006, hereafter M06). Unlike H04, an idealized model initialization was used based on observations from the 1990s that indicated that mesoscale convective vortices (MCVs) forming in the stratiform region, near midlevels of the troposphere in the trough region of tropical disturbances, are often precursors to TC genesis in the deep tropics (e.g. Zehr, 1992; Mapes and Houze, 1995; Harr and Elsberry, 1996; Harr et al., 1996; Bister and Emanuel, 1997; Ritchie and Holland, 1997; Raymond et al., 1998; Reasor et al., 2005). They used the Regional Atmospheric Modeling System (RAMS) to explore the formation of the TC surface circulation through a series of idealized simulations of convection initiated within a preexisting MCV. For the majority of experiments the maximum wind speed of the initial vortex was $6.6 \mathrm{~m} \mathrm{~s}^{-1}$ at a radius of $75 \mathrm{~km}$ and a height of $4 \mathrm{~km}$. The mean Atlantic hurricane season sounding from Jordan (1958) was used to initialize the model thermodynamic structure. The center of the vortex was moistened at low levels and the sea surface temperature was set to a constant value of $29^{\circ} \mathrm{C}$ for most of the experiments. The microphysics scheme included the ice phase, and a radiation scheme was used for all the simulations conducted. An important ramification of this study was that it provided support for the view that a relatively weak mid-level MCV in a favorable thermodynamic environment, over a warm ocean, would gradually undergo a metamorphosis into a TC in the absence of other influences, such as large-scale forcing, or merger of two or more MCVs (e.g. Simpson et al., 1997; Sippel et al., 2006). Factors such as large-scale forcing and merger of MCVs may increase the likelihood of genesis in some situations. However, the M06 study suggested the possibility that an embryonic vortex in an environment devoid of hostile influences such as strong vertical wind shear or dry air intrusion may gradually undergo a transformation into a TC.

Recent work by Dunkerton et al. (2009) broadened the earlier investigation of M06 to include synoptic and subsynoptic-scale influences associated with easterly waves and their embedded critical layers and cyclonically recirculating flow within. Based on their examination of 55 developing cases in the Atlantic and eastern Pacific sectors during the peak of four consecutive hurricane seasons, Dunkerton et al. (2009) developed the new "marsupial paradigm" for tropical cyclogenesis that explicitly recognizes the intrinsic multiscale nature of the problem. The critical layer of a tropical easterly wave, or "wave pouch", was hypothesized to be important to TC formation. The new cyclogenesis model is supported in part by observations of a developing Pacific easterly wave (Montgomery et al., 2010; Raymond and López Carrillo, 2011) and high resolution cloud-representing numerical simulations in both real-case and idealized configurations (Zhang et al., 2011; Montgomery et al., 2010, 2012; Wang et al., 2010). Despite these encouraging scientific results, questions remain about the nature of the convective organization process within the wave pouch in the early stages of a tropical disturbance. Some of the issues are exemplified by the two different cyclogenesis pathways reported in M06 and Nolan (2007, hereafter N07). Unlike M06s simulations where surface winds gradually increased to tropical cyclone strength, N07s simulations showed a strengthening of the mid-level circulation followed by the sudden formation of an intense small scale vortex with strongest winds at the surface. Another open issue concerns the thermodynamic nature of tropical cyclogenesis (Rappin et al., 2010; Raymond et al., 2011; Smith and Montgomery, 2012; Montgomery and Smith, 2012).

Some of the specific issues arising with the two studies of M06 and N07 can be summarized as follows: although M06 found a strengthening of the mid-level circulation often occurred in their simulations (e.g. Fig. 4c of M06), this mid-level strengthening did not appear to play a crucial role in the genesis process. In contrast, N07 found a significantly different pathway to tropical cyclogenesis occurred in simulations conducted with the Weather Research and Forecasting (WRF) model, one in which a mid-level vortex was suggested to play an important role. Some of the experiments in N07 were initialized with a mid-level vortex, similar to M06. The maximum strength of this initial vortex was stronger with a maximum wind speed of $10 \mathrm{~m} \mathrm{~s}^{-1}$ at a radius of $100 \mathrm{~km}$ and a height of $3.72 \mathrm{~km}$. Unlike M06, the initial vortex was not moistened above that of the surrounding environment, and radiation was not included. After approximately three days simulation time, a strengthening and contraction of the mid-level circulation occurred, with azimuthally averaged winds reaching about $12 \mathrm{~m} \mathrm{~s}^{-1}$ at a radius of $65 \mathrm{~km}$ and at a height of $5 \mathrm{~km}$. Low-level winds increased also to approximately $8 \mathrm{~m} \mathrm{~s}^{-1}$. At this time, a small surfaceconcentrated vortex (SSCV) formed suddenly near the center of the large-scale circulation, with a radius of about $7 \mathrm{~km}$, and subsequently the system rapidly developed. N07 emphasized that genesis did not occur until the inner core had achieved deep near saturation and the mid-level vortex had elevated, contracted, and intensified. In the hours before genesis, the intensification of the mid-level vortex was shown to lead to a 
large increase in the (axisymmetric) efficiency of the conversion of latent heat energy to the kinetic energy of the cyclonic wind field, in accordance with the theory of Schubert et al. (1980) and Hack and Schubert (1986). In some respects, this description of the evolution resembles the conceptual model proposed by Bister and Emanuel (1997) who also postulate a crucial role for the mid-level vortex in TC genesis.

The current study presents results of simulations conducted with a newer version of RAMS than that used by M06. Unlike simulations with the previous version of RAMS, the simulations with the newer version show more of a tendency to produce a prominent mid-level vortex. Additionally, the newer version sometimes shows the subsequent formation of a SSCV very reminiscent of the results of N07. In this study we will refer to the pathway described by M06 as pathway One and the pathway described by N07 as pathway Two. Experiments are performed to examine the sensitivity of the pathway taken to model physics and initial conditions. We summarize the distinctive features of these different pathways as follows:

Pathway One: pathway One proceeds by spin up of cyclonic surface winds that become greater than those of the initial weak mid-level vortex and remain stronger than or comparable to the winds aloft, until they become large enough that they reach tropical depression strength (defined herein as approximately $12 \mathrm{~m} \mathrm{~s}^{-1}$ ). Development is characterized by a gradual decrease of the radius of maximum surface winds. Vorticity is concentrated in numerous deep convective towers that collectively drive a system-scale inflow. Vorticity gradually builds in the center as the system-scale inflow produces increasing cyclonic vorticity and as small-scale cyclonic vorticity anomalies are converged at low levels and undergo aggregation. The foregoing process is usually a stochastic process with no prominent event leading to a sudden fall in the minimum surface pressure.

Pathway Two: for pathway Two a mid-level vortex develops in excess of $10 \mathrm{~m} \mathrm{~s}^{-1}$ that is considerably stronger than the typical MCV associated with the stratiform region of a mesoscale convective system (MCS). Its size is usually smaller than the vortex used to initialize the model, with a radius of maximum winds ranging from approximately 25 to $100 \mathrm{~km}$, for the experiments discussed herein. Although the surface winds may strengthen prior to the development of the strong mid-level vortex, with a concomitant decrease in the radius of maximum tangential winds similar to pathway One, the intensification of the surface winds ends once the strong mid-level vortex is established. The system development is then followed by the sudden formation of a SSCV near the center of the larger-scale circulation. The formation of the SSCV is accompanied by a local decrease of a few millibars in the surface pressure. Once formed, the SSCV becomes the focus of a strengthening cyclonic circulation that grows in size, and often develops rapidly into a small TC.

Although the newer version of RAMS behaves differently than the older version in that it is more likely to show devel- opment along pathway Two, we should be careful not to draw definitive conclusions at this stage because results demonstrate a significant sensitivity to model physics and initial conditions. Further refinements to model parameterizations could lead to a different picture of the likelihood of a particular pathway being favored. The solution to this initial value problem appears to have similarities to a bifurcation in that small changes to parameters seem to lead to a sudden qualitative change in the behavior. The strength of the mid-level circulation just prior to genesis for systems developing along pathway Two in the simulations of N07 and those discussed in this paper are notably large, often in excess of $12 \mathrm{~m} \mathrm{~s}^{-1}$. Observations of MCVs that would support the existence of such strong wind speeds prior to genesis are limited. Furthermore, we are unaware of any observed cases that clearly show the abrupt formation of a SSCV.

As far as we are aware, the rather remarkable transformations that take place along pathway Two have been only demonstrated so far by N07 and the results presented in this modeling study. Other numerical modeling studies by H04, M06, Wang et al. (2010) and Braun et al. (2010), do not show development along pathway Two. The initial conditions used in the studies by N07 and herein, are idealized. Large-scale forcing, complex interactions between multiple mesoscale vortices, vertical wind shear, and development within a tropical wave, absent in the idealized model setup, could all have significant influences on the pathway taken to tropical cyclogenesis. Nevertheless, it is interesting that there are two distinct pathways to tropical cyclogensis that are found in numerical modeling simulations even with simple idealized initial conditions, and it is important to understand why this occurs.

As discussed by Montgomery and Smith (2010) the results of N07 raises several questions: what processes lead to the intensification and contraction of the mid-level vortex? Although saturation of the inner core and strengthening of the mid-level vortex occur prior to the formation of the SSCV, are there other factors that influence its development? The small scale vortex intensifies subsequently and grows into the primary circulation, but how this happens requires explanation. N07 considers the formation of the SSCV to be the time of tropical cyclogenesis. Yet, given it is such a small-scale feature, and given the weak larger-scale surface circulation, the classification of the system as a TC at this stage might be considered questionable. Often it is little more than an intense VHT when it forms, although it is characteristically embedded in the center of quite a strong and larger-scale mid-level circulation. Another consideration is that the metamorphosis process described by N07, which is in sharp contrast to the one found in the earlier studies by H04 and M06, may not be the typical pathway along which most TCs form. For our simulations discussed in this paper that evolve along pathway Two, the simulated TC usually remains very small, which suggests that it is unlikely to be the way that most TCs form. N07 also shows results for an initial 
surface vortex case with a radius of maximum winds of $5 \mathrm{~km}$ when the SSCV forms increasing to only $15 \mathrm{~km}$ at hurricane strength (N07 Fig. 3). Nevertheless, it may turn out to be an important mechanism for the formation of small TCs, sometimes referred to as midget TCs, which are hard to forecast. Also, it is important for hurricane modelers and forecasters to be aware that such a pathway may exist, so that they recognize it if it occurs in their simulations or observations. This exploratory study seeks to further our understanding of why a particular pathway may be favored and elucidate some of the factors responsible for evolution along pathway Two.

An outline of the remaining paper is as follows: in Sect. 2, we discuss the numerical model, and the initial conditions used for the experiments. In Sect. 3, results are presented. Simple statistics are compiled for the experiments so that their general behavior can be compared and contrasted. Individual cases are then analyzed in more detail. This is followed by an examination of the transformations that take place during pathway Two, in particular the processes that appear to be important in the development of a prominent mid-level vortex and subsequently the abrupt formation of a SSCV. Results are discussed in Sect. 4 and conclusions are presented in Sect. 5.

\section{Numerical model description and initial conditions}

\subsection{Cloud model}

The model is version 4.3 of RAMS which is more recent than the version used by M06. It is very similar in construct although numerous small changes were made to the code. The main features are that it is a non-hydrostatic numerical modeling system comprising time-dependent equations for velocity, non-dimensional pressure perturbation, ice-liquid water potential temperature (Tripoli and Cotton, 1981), total water mixing ratio and cloud microphysics. The microphysics scheme has categories for cloud droplets, rain, pristine ice crystals, snow, aggregates and hail (Walko, 1995). The surface parameterization of heat, vapor and momentum fluxes is based on the Louis (1979) scheme. The roughness length over water is dependent on the surface wind speed according to the relation derived by Charnock (1955). It includes the radiation scheme developed by Harrington et al. (2000), which is employed for many of the experiments. Long-wave and shortwave components, with diurnal variation, are both activated when the radiation scheme is included. A simpler scheme developed by Chen and Cotton (1987) is an option in RAMS and is used for a sensitivity experiment. Both schemes allow for interactions of the radiation with cloud hydrometeors. A standard first-order sub-grid scale turbulence scheme developed by Smagorinsky (1963) is used with modifications by Lilly (1962) and Hill (1974) that enhance diffusion in unstable conditions and reduces diffusion in stable conditions. RAMS utilizes the two-way interactive multiple nested grid scheme developed by Clark and Farley (1984). The radiative boundary condition described by Klemp and Wilhelmson (1978) is used at the lateral boundary of the coarse grid. A Rayleigh friction layer is included at upper levels. The values of the model parameters employed remain the same as in the previous study (see the appendix of M06), except that the value of Charnock's constant is reduced from 0.018 to 0.016 .

For all the numerical experiments three grids are used. The horizontal grid increments are 24,6 and $2 \mathrm{~km}$, with $(x, y, z)$ dimensions of $101 \times 101 \times 33,110 \times 110 \times 33,161 \times 161 \times$ 33 , respectively, for the small vortex simulations and $101 \times$ $101 \times 33,110 \times 110 \times 33,201 \times 201 \times 33$, respectively, for the large vortex simulations. Each grid is centered within the next coarsest grid. The vertical grid increment is $200 \mathrm{~m}$ and gradually stretched with height to the top of the domain at $z=23.4 \mathrm{~km}$. The depth of the Rayleigh friction layer is $6 \mathrm{~km}$.

\subsection{Initial environmental temperature and moisture profiles}

Similarly to M06, the temperature structure is the mean Atlantic hurricane season sounding of Jordan (1958). The initial moisture profile is the same as that used for the reduced CAPE experiments in M06, referred to as B2 and B3 in that study. This profile has low-level moisture reduced from the Jordan sounding by a maximum of $2 \mathrm{~g} \mathrm{~kg}^{-1}$ at the surface. The reduced moisture of this profile may be more representative of the environment surrounding a TC, which would be expected to have a moister core. It also has the advantage of producing a more focused development, so that there is less convective activity in the coarser grids that only poorly resolve convective scales.

\subsection{Experiments}

The details of the procedure used to initialize the MCV are discussed by M06. Maximum tangential winds for all the experiments are at a height of $4 \mathrm{~km}$ above sea level. Experiments are broadly grouped into the following categories: (1) Radiation versus no radiation. Simulations are conducted either with the Harrington scheme activated or deactivated. (2) Moist versus dry. For most experiments the initial vortex is moistened below $8 \mathrm{~km}$ and for a radii less than the radius of maximum winds (RMW), to $85 \%$ of saturation with respect to liquid. This moisture anomaly is linearly reduced to environmental values from the RMW to a radius of 25 $\mathrm{km}$ beyond the RMW. For the dry simulations there is no vortex moistening and the moisture is horizontally homogeneous at environmental values. (3) Small versus large vortices. Experiments are conducted for two vortex sizes: one having a RMW of $75 \mathrm{~km}$, and one with a RMW of $125 \mathrm{~km}$. (4) Weak versus strong. Most of the experiments are initialized with a relatively weak vortex having a maximum wind speed of $8 \mathrm{~m} \mathrm{~s}^{-1}$ at a height of $4 \mathrm{~km}$ above the surface, and a 
maximum surface wind speed of $4 \mathrm{~m} \mathrm{~s}^{-1}$. A few experiments were carried out with a stronger vortex having a maximum wind speed of $12 \mathrm{~m} \mathrm{~s}^{-1}$ at a height of $4 \mathrm{~km}$ above the surface, and a maximum surface wind speed of $6 \mathrm{~m} \mathrm{~s}^{-1}$. (5) Sea surface temperature (SST). The majority of experiments used a SST of $29^{\circ} \mathrm{C}$. Some simulations were performed with SSTs of $28^{\circ} \mathrm{C}$ and $27^{\circ} \mathrm{C}$.

As an example of an initial vortex, Fig. 1 shows the tangential wind speed, potential temperature perturbation and water vapor mixing ratio for a small weak vortex that has a moisture anomaly. The maximum wind speeds are at a height of $4 \mathrm{~km}$ and a radius of $75 \mathrm{~km}$. The potential temperature anomaly is a maximum at $z=6 \mathrm{~km}$ and a minimum at $z=2 \mathrm{~km}$ at the center of the vortex. Moistening to $85 \%$ of saturation below $8 \mathrm{~km}$ within the vortex increases the water vapor mixing ratio by approximately $2 \mathrm{~g} \mathrm{~kg}^{-1}$ relative to the environment. The effects of the moisture anomaly are included in the buoyancy and virtual potential temperature used to specify the initial vortex (M06), so that it remains well balanced. These changes are reflected in the potential temperature perturbation field and are particularly evident where the horizontal gradient of water vapor is strong between $75-100 \mathrm{~km}$. The saturated vapor pressure is a function of temperature that is perturbed in the vortex resulting in a water vapor mixing ratio field that is not completely flat in regions that are $85 \%$ of saturation.

Table 1 shows 16 experiments numbered 1 to 16 conducted using the grouping discussed above. All of these experiments have ice microphysics except Experiment 1 that is the only simulation that does not have ice microphysics. This simpler microphysics case is discussed first. It is identical to Experiment 2 except it only has cloud water and rain microphysical categories. Experiments 2 to 7 do not have radiation. These simpler physics cases are discussed prior to Experiments 8 to 16 that include radiation. Additionally, one more simulation, Experiment 17, was conducted using the Chen-Cotton radiation scheme instead of the Harrington scheme.

Experiment 2 is similar to the reduced CAPE simulations B2 and B3 of M06 except that the form of the moist anomaly is different and radiation is not included. Experiment 3 is identical to Experiment 2 except the initial vortex is dry. Experiment 4 is similar to 2 except the initial vortex is large. Experiments 5 and 6 have a lower SST of $28^{\circ} \mathrm{C}$ for a small and large vortex, respectively. Experiment 7 is identical to Experiment 2 except the SST is reduced from 29 to $27^{\circ} \mathrm{C}$. Experiment 8 is identical to Experiment 2 except that radiation is activated. Experiment 9 is identical to 8 except the initial vortex is dry. Experiments 10 and 11 are stronger vortex cases for moist and dry small vortices, respectively. Experiment 12 is identical to 8 except the vortex is large. Experiment 13 is identical to Experiment 12 except the vortex is strong. Experiments 14-16 examine the effects of reducing SST when radiation is activated. Finally, Experiment 17 is identical to Experiment 8 except that the Chen-Cotton radiation scheme is employed. Clearly, many more combinations are possible.
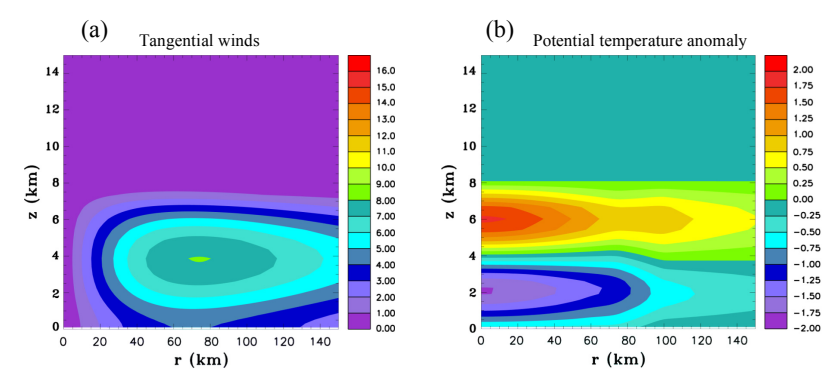

(c) Vapor mixing ratio

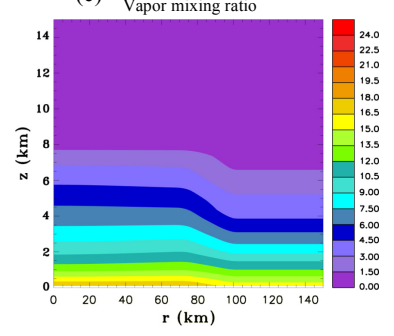

Fig. 1. Initial conditions for a small weak vortex with a moisture anomaly. (a) Tangential winds. The contour interval is $1 \mathrm{~m} \mathrm{~s}^{-1}$. (b) Potential temperature anomaly. The contour interval is $0.25 \mathrm{~K}$. (c) Water vapor mixing ratio. The contour interval is $1.5 \mathrm{~g} \mathrm{~kg}^{-1}$.

The experiments that have been chosen should give a fairly comprehensive view of the affects of changing some of the major parameters on the pathway taken to tropical cyclogenesis.

\section{Experimental results}

\subsection{Discussion of the general behavior}

Table 2 shows some general statistics for the experiments that include the time at which the maximum averaged tangential wind at the grid point adjacent to the surface $(z=98 \mathrm{~m})$ reaches $12 \mathrm{~m} \mathrm{~s}^{-1}$, and the RMW at this time. For systems developing along pathway One, this wind speed is a reasonable criterion for concluding that tropical cyclogenesis has occurred. For systems developing along pathway Two that form an SSCV, this wind speed is probably not a good indicator of genesis since the larger-scale circulation at the surface is still weak. Also shown is the pathway taken to genesis. Designation as pathway Two requires the development of a prominent mid-level vortex and the subsequent abrupt formation of a resilient SSCV that becomes the focus of a developing TC. Experiments 11 and 13 actually developed a smallscale vortex but it was not resilient and instead merged with the larger-scale circulation. These cases are therefore designated as developing along pathway One. Two experiments, 14 and 16, first developed into TCs along pathway One, but subsequently weakened and later underwent a second genesis along pathway Two. These are therefore designated $1 / 2$ in the table. Also shown for the pathway Two cases is the maximum 
Table 1. Experiments $1-16$ categorized by either no radiation, or radiation included; moist, or dry; small, or large; weak, or strong; value of SST is either 29,28 , or $27^{\circ} \mathrm{C}$.

\begin{tabular}{lccccccccccc}
\hline Exp. & no radiation & radiation & moist & dry & small & large & weak & strong & SST 29 & SST 28 & SST27 \\
\hline $1^{*}, 2$ & $\otimes$ & & $\otimes$ & & $\otimes$ & & $\otimes$ & & $\otimes$ & & \\
3 & $\otimes$ & & & $\otimes$ & $\otimes$ & & $\otimes$ & & $\otimes$ & & \\
4 & $\otimes$ & & $\otimes$ & & & $\otimes$ & $\otimes$ & & $\otimes$ & & \\
5 & $\otimes$ & & $\otimes$ & & $\otimes$ & & $\otimes$ & & & $\otimes$ & \\
6 & $\otimes$ & & $\otimes$ & & & $\otimes$ & $\otimes$ & & & $\otimes$ & \\
7 & $\otimes$ & & $\otimes$ & & $\otimes$ & & $\otimes$ & & & & $\otimes$ \\
$8,17^{* *}$ & & $\otimes$ & $\otimes$ & & $\otimes$ & & $\otimes$ & & $\otimes$ & & \\
9 & & $\otimes$ & & $\otimes$ & $\otimes$ & & $\otimes$ & & $\otimes$ & & \\
10 & & $\otimes$ & $\otimes$ & & $\otimes$ & & & $\otimes$ & $\otimes$ & & \\
11 & & $\otimes$ & & $\otimes$ & $\otimes$ & & & $\otimes$ & $\otimes$ & & \\
12 & & $\otimes$ & $\otimes$ & & & $\otimes$ & $\otimes$ & & $\otimes$ & & \\
13 & & $\otimes$ & $\otimes$ & & & $\otimes$ & & $\otimes$ & $\otimes$ & & \\
14 & & $\otimes$ & $\otimes$ & & $\otimes$ & & $\otimes$ & & & $\otimes$ & \\
15 & & $\otimes$ & $\otimes$ & & & $\otimes$ & $\otimes$ & & & $\otimes$ & \\
16 & & $\otimes$ & $\otimes$ & & $\otimes$ & & $\otimes$ & & & & $\otimes$ \\
\hline
\end{tabular}

* Experiment 1 is the only experiment without ice physics.

** Experiment 17 uses the Chen-Cotton radiation scheme.

Table 2. General statistics for the experiments: shown are the time the maximum azimuthally averaged tangential wind speeds near the surface reach $12 \mathrm{~m} \mathrm{~s}^{-1}$; the near-surface radius of maximum winds (RMW) at this time; the pathway taken to genesis; the maximum nearsurface tangential winds prior to the SSCV forming for systems that develop along pathway Two; the time at which the system becomes a tropical storm; the RMW at this time; the time the system becomes a hurricane; the RMW at this time.

\begin{tabular}{|c|c|c|c|c|c|c|c|c|c|}
\hline Exp. & Description & $\begin{array}{l}T_{12} \\
\text { (h) }\end{array}$ & $\begin{array}{l}\mathrm{RMW}_{12} \\
(\mathrm{~km})\end{array}$ & Path & $\begin{array}{l}V_{\mathrm{Max}} \text { prior } \\
\text { to } \operatorname{SSCV}\left(\mathrm{m} \mathrm{s}^{-1}\right)\end{array}$ & $\begin{array}{l}T_{\mathrm{TS}} \\
\text { (h) }\end{array}$ & $\begin{array}{l}\mathrm{RMW}_{\mathrm{T}} \\
(\mathrm{km})\end{array}$ & $\begin{array}{l}T_{\mathrm{H}} \\
(\mathrm{h})\end{array}$ & $\begin{array}{l}\mathrm{RMW}_{\mathrm{H}} \\
(\mathrm{km})\end{array}$ \\
\hline 1 & No ice, no radiation, moist, small, weak, SST29 & 39 & 15 & 1 & & 41 & 15 & 48 & 15 \\
\hline 2 & No radiation, moist, small, weak, SST29 & 82 & 9 & 2 & 10.2 & 92 & 13 & 103 & 13 \\
\hline 3 & No radiation, dry, small, weak, SST 29 & 151 & 5 & 2 & 6.4 & 174 & 11 & 189 & 13 \\
\hline 4 & No radiation, moist, large, weak, SST29 & 89 & 5 & 2 & 10.0 & 103 & 23 & 114 & 23 \\
\hline 5 & No radiation, moist, small, weak, SST28 & 95 & 17 & 1 & & 101 & 11 & 110 & 11 \\
\hline 6 & No radiation, moist, large, weak, SST28 & 112 & 41 & 1 & & 118 & 37 & 127 & 23 \\
\hline 7 & No radiation, moist, small, weak, SST27 & 86 & 37 & 1 & & 126 & 23 & 146 & 15 \\
\hline 8 & Radiation, moist, small, weak, SST29 & 60 & 5 & 2 & 9.7 & 65 & 11 & 78 & 13 \\
\hline 9 & Radiation, dry, small, weak, SST29 & 101 & 11 & 2 & 10.2 & 105 & 13 & 119 & 15 \\
\hline 10 & Radiation, moist, small, strong, SST29 & 29 & 61 & 1 & & 43 & 15 & 48 & 17 \\
\hline 11 & Radiation, dry, small, strong, SST29 & 45 & 57 & 1 & & 58 & 15 & 64 & 15 \\
\hline 12 & Radiation, moist, large, weak, SST29 & 55 & 5 & 2 & 9.3 & 61 & 19 & 69 & 15 \\
\hline 13 & Radiation, moist, large, strong, SST29 & 35 & 87 & 1 & & 47 & 53 & 54 & 31 \\
\hline 14 & Radiation, moist, small, weak, SST28 & 37 & 19 & $1 / 2$ & & 68 & 9 & 75 & 9 \\
\hline 15 & Radiation, moist, large, weak, SST28 & 49 & 5 & 2 & 9.4 & 52 & 9 & 68 & 15 \\
\hline 16 & Radiation, moist, small, weak, SST27 & 41 & 15 & $1 / 2$ & & 77 & 11 & 82 & 7 \\
\hline 17 & Chen radiation, moist, small, weak, SST29 & 20 & 39 & 1 & & 37 & 41 & 47 & 21 \\
\hline
\end{tabular}

averaged tangential wind at the lowest model level that occurs in the period before the SSCV forms. The next columns give the time at which the TC develops into a tropical storm $\left(T_{\mathrm{TS}}\right)$ using the criteria that the average tangential winds at the lowest model level reach $17.4 \mathrm{~m} \mathrm{~s}^{-1}$, the RMW at this time, the time at which the TC reaches hurricane strength $\left(T_{\mathrm{H}}\right)$ using a criteria of $33 \mathrm{~m} \mathrm{~s}^{-1}$ and the RMW at this time.

From this table the following conclusions can be drawn: (1) both pathways are about equally represented in this set of experiments. (2) The moist cases develop much more rapidly than the dry cases given that the other parameters are the same. (3) Radiation tends to produce faster development. (4) Pathway One is favored if the initial vortex is strong or if the sea surface temperatures are lower. (5) A significant fraction of the systems that develop along pathway Two reach wind speeds of approximately $10 \mathrm{~m} \mathrm{~s}^{-1}$ near the surface prior to an SSCV forming, so they are already a borderline tropical depression. (6) The SSCVs that form for systems developing 
Table 3. Properties of the prominent mid-level vortex (MLV) that form for systems that develop along pathway Two: shown are the maximum azimuthally averaged tangential velocities of the MLV, its RMW and height at the time the SSCV forms; the time the SSCV forms; the maximum near-surface tangential winds and the near-surface RMW, at this time.

\begin{tabular}{|c|c|c|c|c|c|c|c|}
\hline Exp. & Description & $\begin{array}{l}\text { MLV } \\
V_{\max }\left(\mathrm{m} \mathrm{s}^{-1}\right)\end{array}$ & $\begin{array}{l}\text { MLV } \\
\text { RMW (km) }\end{array}$ & $\begin{array}{l}\text { MLV } \\
\text { height }(\mathrm{km})\end{array}$ & $\begin{array}{l}\text { SSCV } \\
T_{\text {form }}(\mathrm{h})\end{array}$ & $\begin{array}{l}V_{\max } \text { at } \\
\text { surface }\left(\mathrm{m} \mathrm{s}^{-1}\right)\end{array}$ & $\begin{array}{l}\text { RMW at } \\
\text { surface }(\mathrm{km})\end{array}$ \\
\hline 2 & No radiation, moist, small, weak, SST29 & 13.9 & 29 & 4.9 & 80 & 8.3 & 61 \\
\hline 3 & No radiation, dry, small, weak, SST29 & 12.3 & 63 & 4.9 & 147 & 5.1 & 147 \\
\hline 4 & No radiation, moist, large, weak, SST29 & 15.3 & 75 & 5.4 & 88 & 9.3 & 103 \\
\hline 8 & Radiation, moist, small, weak, SST29 & 12.5 & 41 & 5.4 & 58 & 6.8 & 47 \\
\hline 9 & Radiation, dry, small, weak, SST29 & 13.7 & 91 & 4.9 & 99 & 2.8 & 127 \\
\hline 12 & Radiation, moist, large, weak, SST29 & 19.1 & 71 & 5.4 & 54 & 9.0 & 96 \\
\hline 14 & Radiation, moist, small, weak, SST28 & 16.3 & 23 & 4.9 & 66 & 8.5 & 25 \\
\hline 15 & Radiation, moist, large, weak, SST28 & 13.5 & 47 & 4.3 & 48 & 7.9 & 69 \\
\hline 16 & Radiation, moist, small, weak, SST27 & 12.9 & 23 & 4.9 & 71 & 7.8 & 25 \\
\hline
\end{tabular}

along pathway Two have a very small RMW and they typically develop very rapidly into tropical storms and hurricanes undergoing a modest radial expansion, or growth, during this period. On the other hand, systems developing along pathway One tend to have a larger RMW and undergo a contraction as they develop. Some of these cases also develop rapidly. (7) Not unexpectedly, larger initial vortices tend to produce larger TCs. (8) Unexpectedly, lower SSTs do not always result in a slower rate of development.

For systems that develop along pathway Two, Table 3 shows properties of the "second mid-level vortex" at the time of formation of the SSCV and the maximum wind speeds and radius of maximum winds at the surface. For these RAMS simulations, we refer to the strong mid-level vortex that forms as the "second mid-level vortex" since the original MCV became less evident as the low-level winds increased during the early evolution of the system. The second midlevel vortex develops winds between $12-16 \mathrm{~m} \mathrm{~s}^{-1}$ for most cases with the exception of Experiment 12 that has winds of $19.1 \mathrm{~m} \mathrm{~s}^{-1}$. The RMW of the mid-level vortex tends to be relatively large for the dry cases and the large initial vortex cases, ranging from 47 to $91 \mathrm{~km}$. The experiments with small initial vortices and lower SSTs develop notably smaller second mid-level vortices. They are centered at a height of approximately $4.3-5.4 \mathrm{~km}$. Comparing Table 2 with Table 3 it can be seen that the SSCVs attain a tangential wind speed of $12 \mathrm{~m} \mathrm{~s}^{-1}$ within a few hours of forming. Surface winds tend to decrease from their maximum values shown in Table 2 as the mid-level vortex develops. Surface winds at the time the SSCV forms for the dry cases are particularly weak. The RMW of the surface winds at this time tends to be significantly larger than the RMW of the mid-level vortex. It is particularly large for the dry cases.

Figure $2 \mathrm{a}$ and $\mathrm{b}$ show examples of the vertical vorticity at the lowest model level adjacent to the surface $(z=98 \mathrm{~m})$ at different stages of development for cases that develop along pathway One and cases that develop along pathway Two, comparing two experiments that are initialized with a small vortex, and two experiments that are initialized with a large vortex. Shown are an early stage prior to tropical cyclogenesis, and stages when mean tangential winds near the surface reach $12 \mathrm{~m} \mathrm{~s}^{-1}$ and $17 \mathrm{~m} \mathrm{~s}^{-1}$. These latter two stages are approximately when tropical cyclonesis can be considered to have occurred and when the tropical cyclone has reached tropical storm strength, respectively. The cases that develop along pathway Two show a very small vortex near the center when winds speed reach $12 \mathrm{~m} \mathrm{~s}^{-1}$, which are the SSCVs.

\subsection{Discussion of individual cases}

Experiment 1: this experiment has the simplest microphysics with only the cloud water and rain categories activated. There is no radiation scheme and the initial vortex is small, weak and has a moisture anomaly. Figure $3 \mathrm{a}-\mathrm{c}$ show time series of the minimum surface pressure, the maximum azimuthally averaged tangential wind and the height it occurs, and the maximum azimuthally averaged tangential wind at the model level adjacent to the surface $(z=98 \mathrm{~m})$ and the radius it occurs, respectively. The minimum surface pressure shows a gradual decrease during the first $35 \mathrm{~h}$ followed by a substantial fall during the next $15 \mathrm{~h}$. Genesis occurs at approximately $t=39 \mathrm{~h}$. The height of the maximum tangential winds gradually falls after $12 \mathrm{~h}$ becoming close to the surface at the time of genesis. Near-surface winds started to increase significantly after $30 \mathrm{~h}$. There is a significant contraction of the radius of maximum winds after $30 \mathrm{~h}$ to approximately $15 \mathrm{~km}$ at genesis. It remains fairly constant during the next $12 \mathrm{~h}$ while the winds rapidly increase to hurricane strength. For this case a second mid-level vortex did not form. The system clearly developed into a tropical cyclone along pathway One.

Experiment 2: this case is identical to Experiment 1 except that ice physics is included. The rate of development was considerably slower. Figure 4 shows that during the first twenty four hours the maximum winds shifted to near the surface with speeds reaching in excess of $10 \mathrm{~m} \mathrm{~s}^{-1}$. A second prominent mid-level vortex developed after $60 \mathrm{~h}$. The sudden formation of a SSCV with a radius of $5 \mathrm{~km}$ is evident in Fig. $4 \mathrm{c}$ at $t=80 \mathrm{~h}$, which was concurrent with a surface 

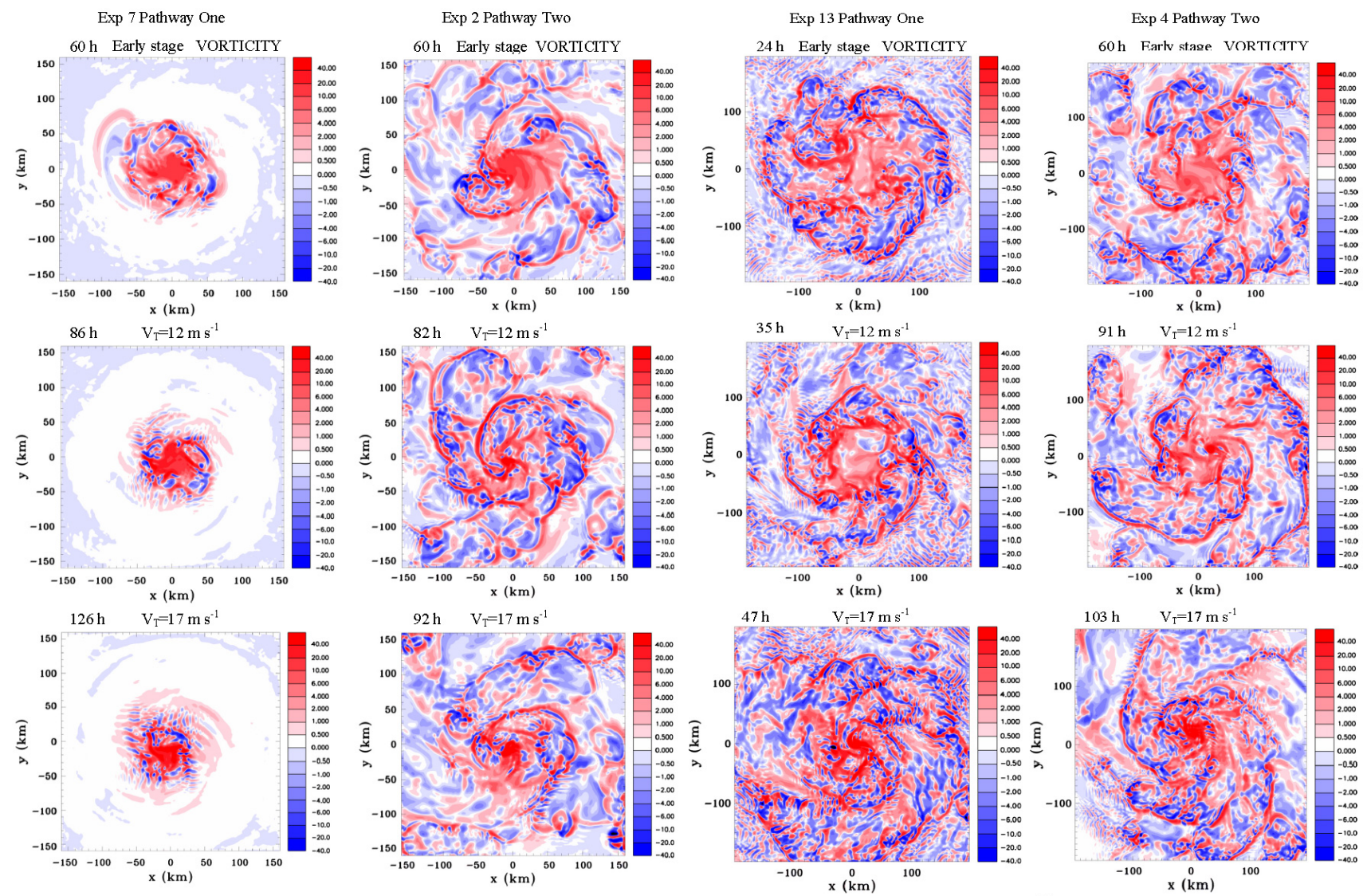

(a)
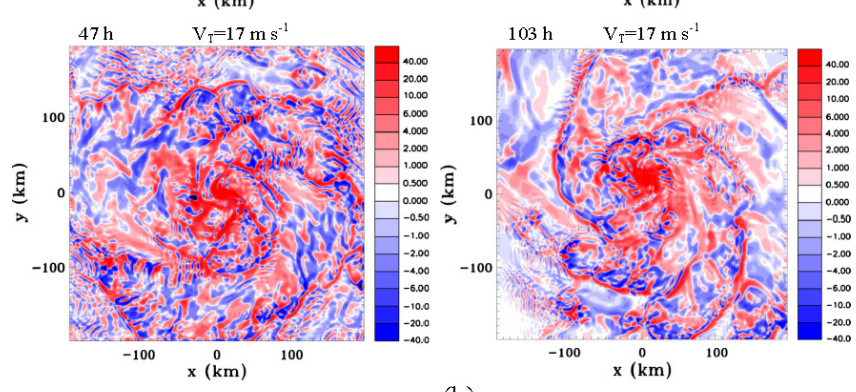

(b)

Fig. 2. Examples of development along pathway One and pathway Two, showing vertical vorticity $\left(\times 10^{-4} \mathrm{rad} \mathrm{s}^{-1}\right)$, at $z=98 \mathrm{~m}$. (a) Experiments 7 and 2 that were initialized with a small vortex, and (b) Experiments 13 and 4 that were initialized with a large vortex. $V_{\mathrm{T}}$ is the azimuthally averaged tangential velocity near the surface.

pressure fall of $\sim 2 \mathrm{mb}$. Near-surface tangential winds of the SSCV reach $12 \mathrm{~m} \mathrm{~s}^{-1}$ a couple of hours later. The small vortex increased in size fairly soon after forming although the system remained relatively small. Figure $5 \mathrm{a}$ and $\mathrm{b}$ show vertical cross sections of the averaged tangential component of the horizontal velocity at $t=79$ and $83 \mathrm{~h}$, respectively. Just prior to the formation of the small vortex the mid-level vortex is centered at approximately $5 \mathrm{~km}$ above the surface and the maximum wind speed is $\sim 12 \mathrm{~m} \mathrm{~s}^{-1}$ at a radius of $\sim 35 \mathrm{~km}$. The strongest surface winds are at a radius of $\sim 60 \mathrm{~km}$. A few hours later a SSCV has formed with near-surface wind speeds in excess of $12 \mathrm{~m} \mathrm{~s}^{-1}$. During this period and afterwards the mid-level vortex continued to strengthen as shown in Fig. $4 \mathrm{~b}$. At $\sim 92 \mathrm{~h}$ the near-surface winds become stronger than the winds aloft. By this time they are already at tropical storm strength. This experiment clearly demonstrates the importance of the ice phase in the development of a second stronger mid-level vortex and the subsequent formation of a SSCV.

Experiment 3: this case is the same as Experiment 2 except there is no initial moisture anomaly. It is similar to an experiment conducted by N07 that also had no moisture anomaly or radiation. In this experiment the environment has less low-level moisture than used in the experiments conducted by N07 since the Jordan sounding was modified as discussed in Sect. 2.2. Figure 6 shows that the initial rate of development was extremely slow for this case. A SSCV formed at $t=147 \mathrm{~h}$ and a pressure fall of $\sim 3 \mathrm{mb}$ occurred in the next few hours. However, the fall in minimum surface pressure was only temporary. Also, the nearsurface wind speeds decreased to less than $10 \mathrm{~m} \mathrm{~s}^{-1}$ after their initial spike to $18 \mathrm{~m} \mathrm{~s}^{-1}$. Sustained development did not occur until after $160 \mathrm{~h}$. The mid-level vortex was considerably larger than for Experiment 2 and appears to be related to the more widespread distribution of deep convection for this case, whereas the presence of an initial vortex moisture anomaly for Experiment 2 resulted in convection being more focused in the center. Figure 7 shows the average relative humidity for a circular area with radius of $100 \mathrm{~km}$ from the vortex center at $z=2.6,4.9$ and $7.3 \mathrm{~km}$. The vortex moistens at low levels first and then more gradually at upper levels due to vertical transport of moisture in deep convective towers. 
(a)

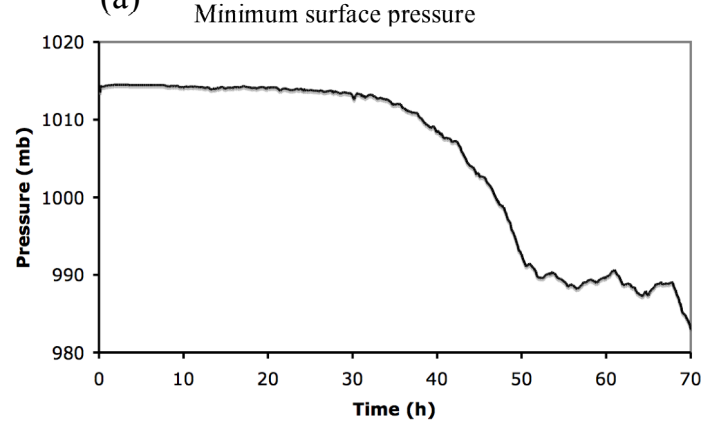

(b)

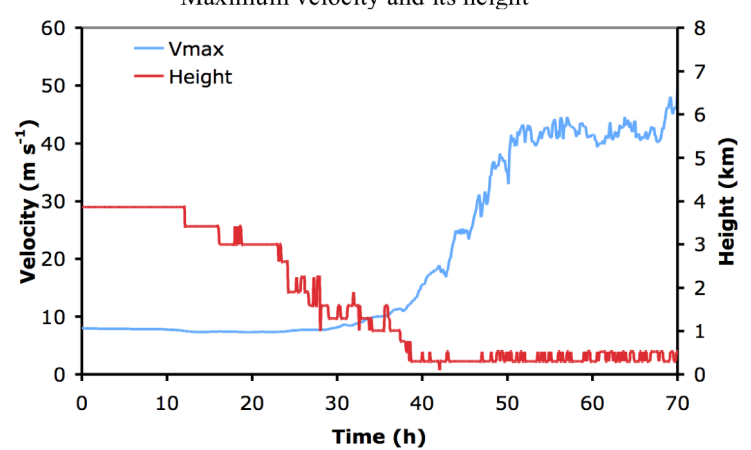

(c)

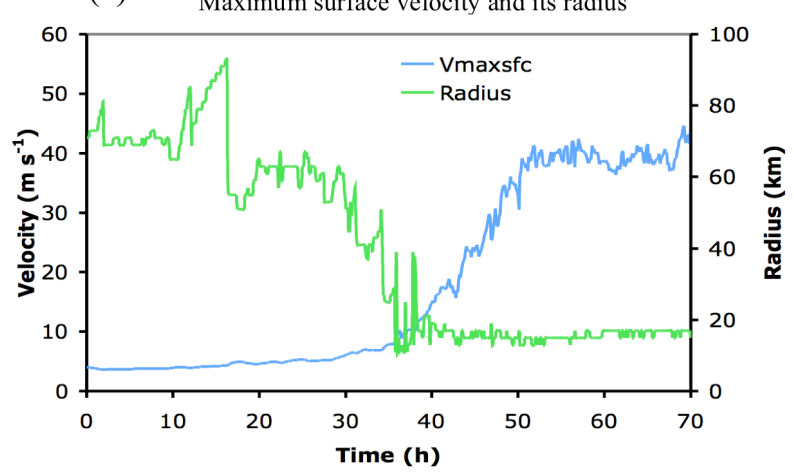

Fig. 3. Time series for Experiment 1, the no ice case. (a) The minimum surface pressure. (b) The azimuthally averaged maximum tangential velocity and the height it occurs. (c) The azimuthally averaged tangential velocity at the lowest model level and the radius it occurs.

At approximately $t=110 \mathrm{~h}$ all three levels show moistening to about $85 \%$ of saturation. Subsequently, the moistening increases at $z=7.3 \mathrm{~km}$, remains approximately constant at $z=4.9 \mathrm{~km}$ and decreases slightly at $2.6 \mathrm{~km}$. It appears that deep moistening was an important prerequisite for genesis, however it is interesting that genesis did not occur until a considerable period of time after high values of relative humidity had been reached at these levels. This case also showed less spin up of low-level winds prior to the development of a second mid-level vortex than Experiment 2. This is consistent with the results of N07 who found low-level winds (a) Minimum surface pressure

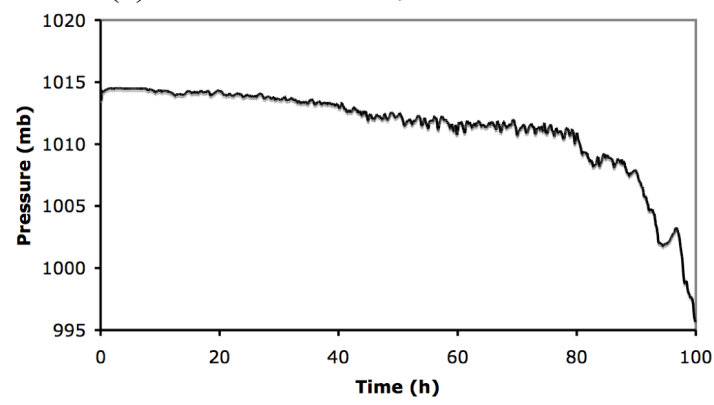

(b)

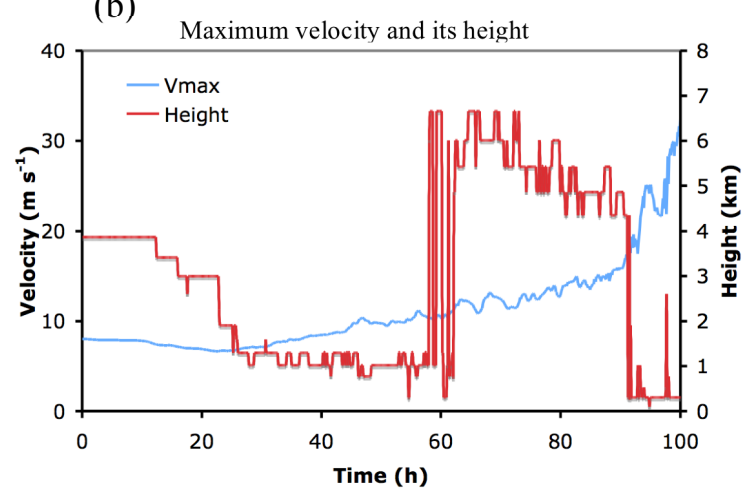

(c)

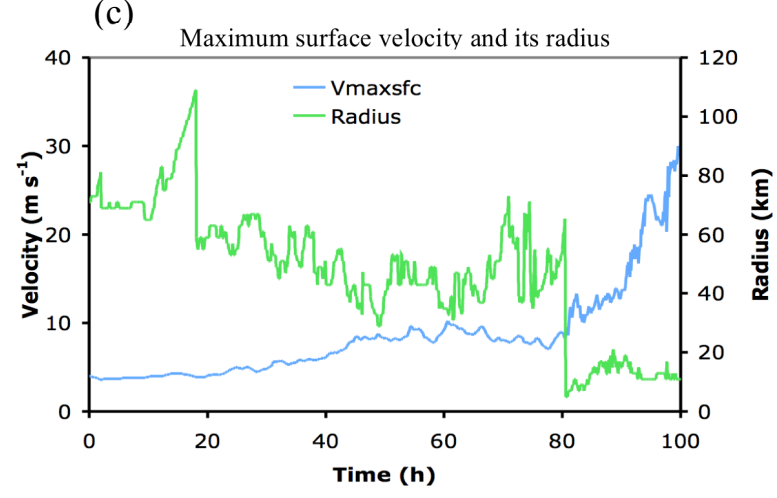

Fig. 4. As in Fig. 2, for Experiment 2.

did not increase significantly for his experiments that did not include an initial low-level moisture anomaly.

Experiment 4: this case is similar to Experiment 2 except that the size of the initial vortex and its associated moisture anomaly is larger. Development was similar in many respects to Experiment 2, but slightly slower. Additionally, it eventually developed a significantly larger tropical storm and hurricane than for Experiment 2. Although a SSCV formed, it grew substantially in size as the system intensified (Table 2). Why this system evolved into a larger hurricane than other systems developing along pathway Two is not certain, but it did develop quite a significant large-scale surface circulation prior to the SSCV forming. Convergence of this large-scale low-level cyclonic vorticity may have been a factor influencing the ultimate size of the circulation at hurricane strength. 
(a)

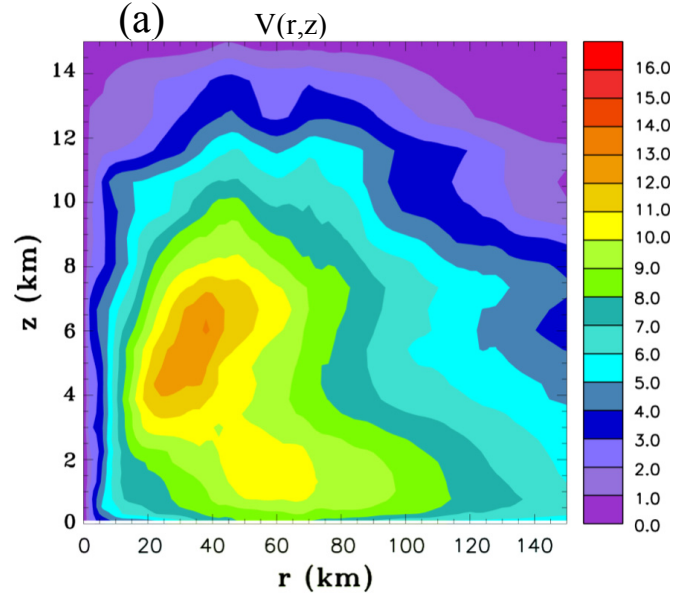

(b) $\quad \mathrm{V}(\mathrm{r}, \mathrm{z})$

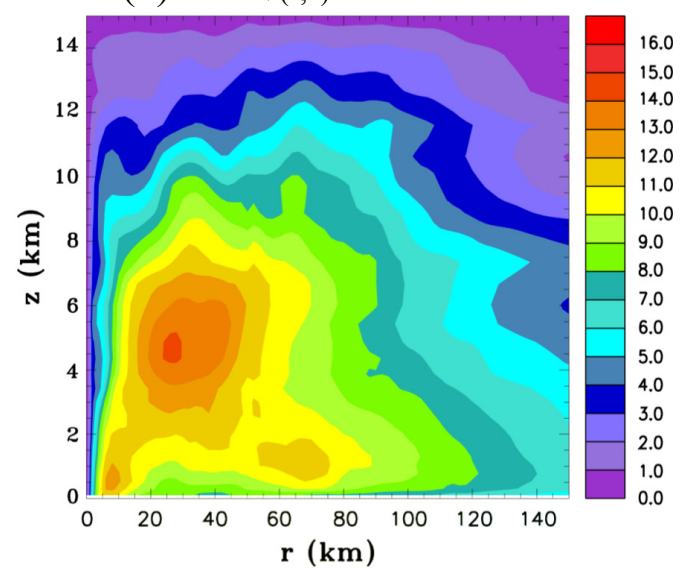

Fig. 5. Azimuthally averaged tangential velocity $V(r, z)$ for Experiment 2 , at (a) $t=79 \mathrm{~h}$, and (b) $t=83 \mathrm{~h}$. The contour interval is $1 \mathrm{~m} \mathrm{~s}^{-1}$.

Experiment 5: this case is similar to Experiment 2 except that the SST is reduced from 29 to $28^{\circ} \mathrm{C}$. Figure 8 shows genesis occurring at approximately $t=95 \mathrm{~h}$. The pathway to genesis is different than for Experiment 2. A significant second mid-level vortex still forms although it is smaller since early convection is more focused near the center for this case (not shown). There is a more gradual decrease in the radius of maximum winds near the surface rather than a clear jump as occurred in Experiment 2 when the very small vortex formed. When the radius of maximum winds near the surface reach $\sim 15 \mathrm{~km}$, wind speeds reach tropical cyclone strength. This is in contrast to Experiments 2, 3 and 4, which show the sudden formation of a very small vortex with a radius of 5-6 km prior to genesis. Although genesis occurs along pathway One for this case, it is clearly very close to a transition point whereby a small increase in SST would likely result in development along pathway Two.

Experiment 6: this case is a larger vortex case similar to Experiment 4 except that the SST is reduced to $28^{\circ} \mathrm{C}$. This (a)

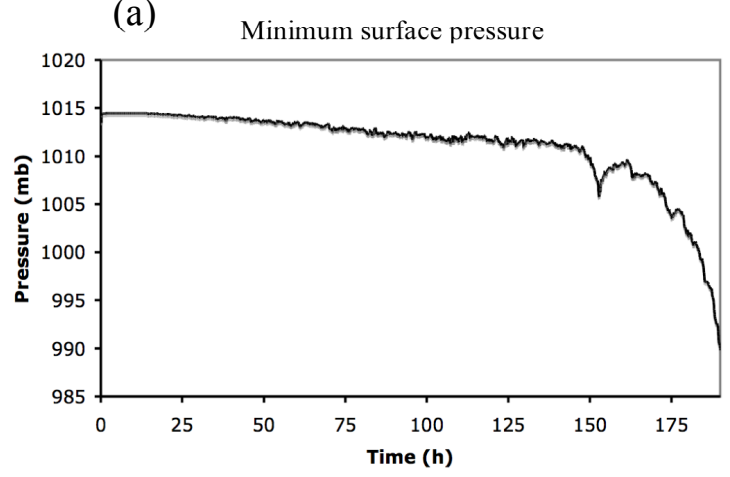

(b)

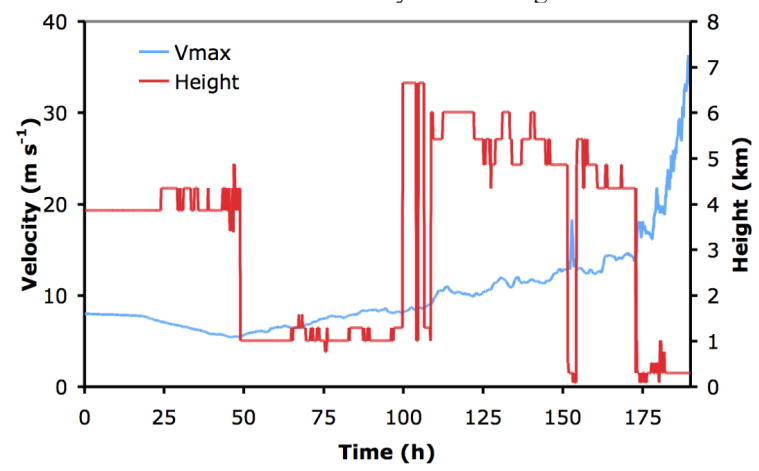

(c) Maximum surface velocity and its radius

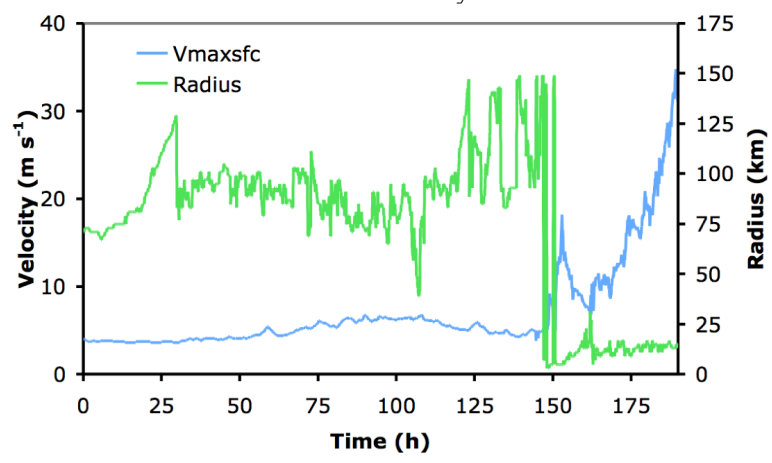

Fig. 6. As in Fig. 2, for Experiment 3.

case underwent genesis at approximately $t=112 \mathrm{~h}$ as shown in Fig. 9. At this time a very small region of strong vorticity developed near the center accompanied by a local pressure fall. This feature, evident in Fig. 8c, was not persistent and only lasted about an hour. This case is therefore classified as developing along pathway One. The radius of maximum winds near the surface, when winds reach tropical cyclone strength, varies considerably with an average of $\sim 40 \mathrm{~km}$. This case also developed a second mid-level vortex prior to genesis. This case is similar to Experiment 5 in that it is close to developing along pathway Two.

Experiment 7: this case is similar to Experiment 5 except that the SST is reduced further to $27^{\circ} \mathrm{C}$. Figure 10 shows that genesis occurs at approximately $t=86 \mathrm{~h}$. This case did not 


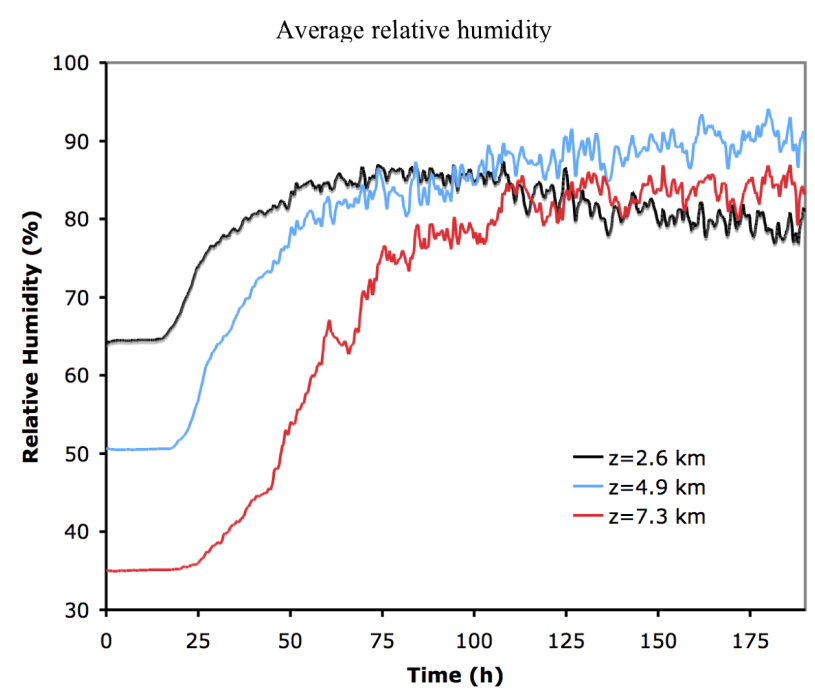

Fig. 7. The average relative humidity for a circular area with radius of $100 \mathrm{~km}$ from the vortex center, at levels $z=2.6,4.9$ and $7.3 \mathrm{~km}$, for Experiment 3.

develop a second mid-level vortex prior to genesis. Later as the system developed into a tropical storm it can be seen in Fig. 10b that mid-level winds were often stronger than those near the surface. Even though the system developed along pathway One it still formed a very small hurricane comparable to many of those that developed along pathway Two.

\subsection{Simulations with a radiation scheme}

In this section, we discuss simulations with the radiation scheme activated, in particular drawing attention to differences with the non-radiation cases discussed previously. Experiment 8 is similar to Experiment 2 except that the Harrington radiation scheme is included. As can be seen in Table 2 this led to a significantly faster rate of genesis. This is true in general for simulations that include radiation compared to similar simulations that do not. The effects of radiation on tropical oceanic systems can broadly be classified as being due to (1) clear sky infrared (IR) cooling, (2) direct radiation-convection interaction (e.g. Randall et al., 1991), and (3) cloud-cloud free radiation difference (Gray and Jacobson, 1977). Clear sky IR cooling is very evident in the environment of the simulations with the radiation scheme included. The strongest IR cooling occurs at $9-10 \mathrm{~km}$ above the surface, which is consistent with the results of Zhang and Chou (1999). This will increase the convective available potential energy (CAPE) over time promoting moist convection. This is probably a significant factor responsible for the faster rate of genesis for the cases with radiation. IR cooling also causes the environmental profile to become closer to saturation, which may also promote the earlier development of convection. Another aspect of the IR cooling is that it leads

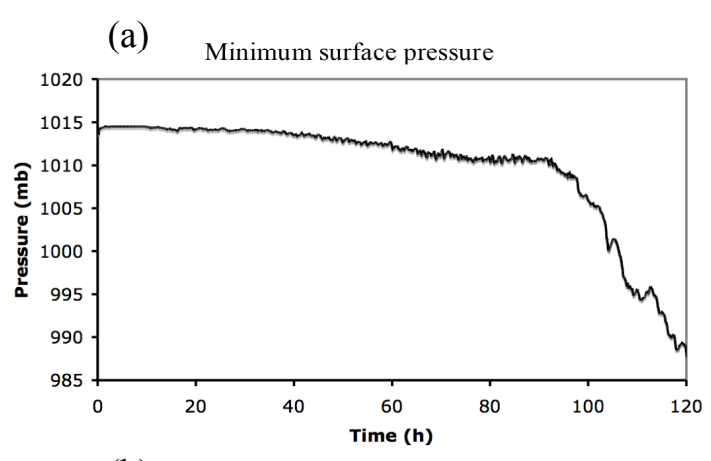

(b)

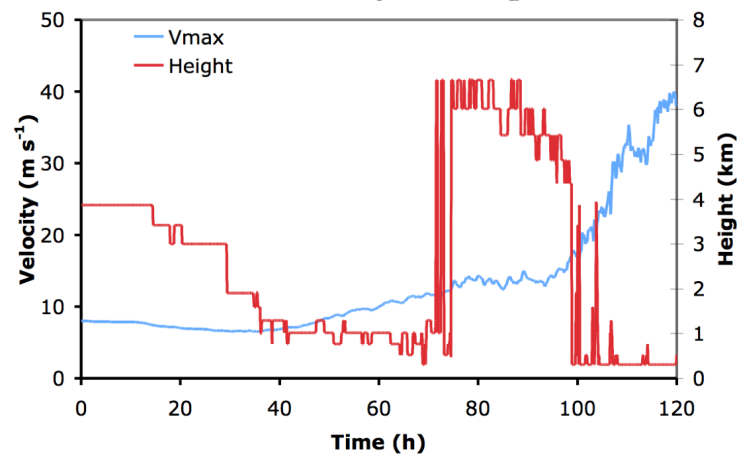

(c)

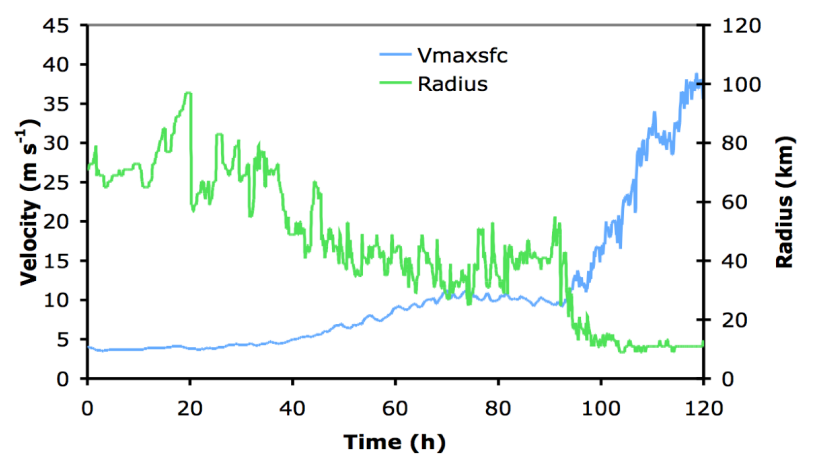

Fig. 8. As in Fig. 2, for Experiment 5.

to an increase in the surface pressure in the environment and also in the core of the system early on when latent heating due to deep convection is relatively small. This is particularly evident in the dry simulations since it takes longer for deep convection to develop.

Experiment 15 is an interesting case since it develops along pathway Two, whereas Experiment 6, which is identical except for the absence of radiation, develops along pathway One. Figure 11 illustrates this development and includes time series of total liquid and ice water within a cylindrical volume with a radius of $100 \mathrm{~km}$ from the center as well as clear sky shortwave radiation at the surface. For the radiation case the minimum surface pressure increases initially due to IR cooling as discussed earlier, in contrast to the non-radiation case that exhibits a decrease. By $t=80 \mathrm{~h}$ the 
(a)

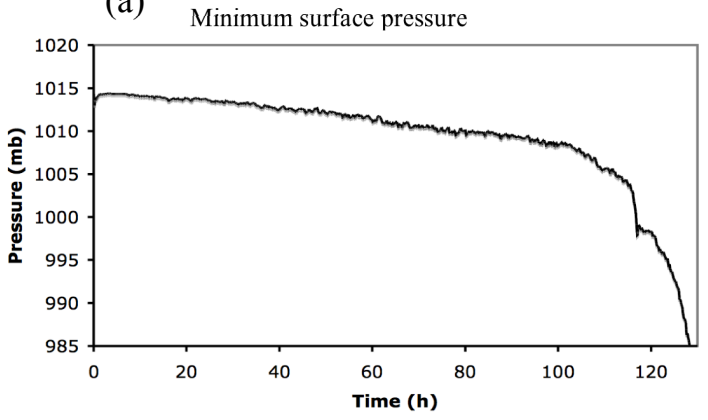

(b)

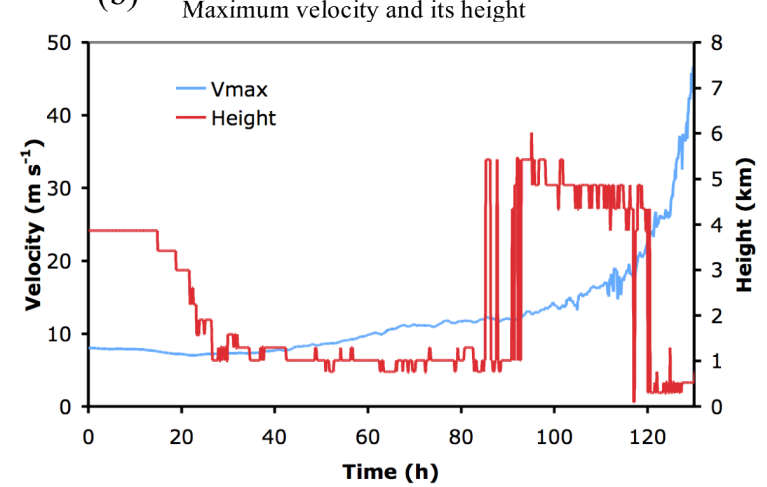

(c) Maximum surface velocity and its radius

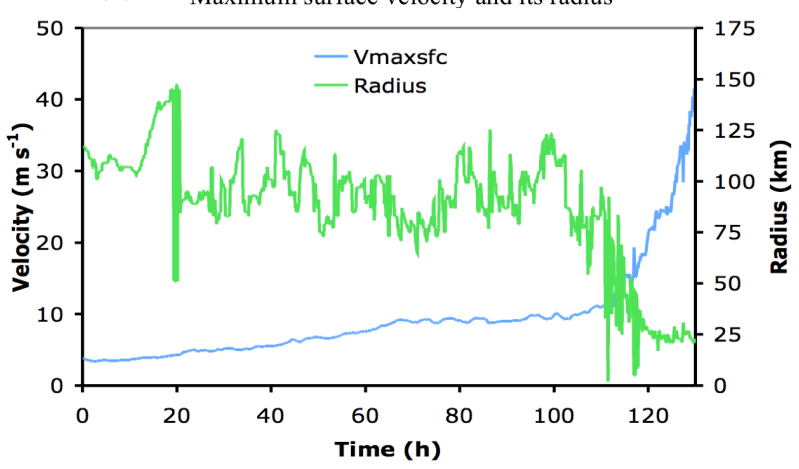

Fig. 9. As in Fig. 2, for Experiment 6.

radiation case has developed into a moderately strong hurricane, whereas the case without radiation does not undergo genesis until approximately $t=112 \mathrm{~h}$ (Fig. 9). Figure 11d shows that the liquid and ice content is far larger for the radiation case. There are large pulses in convective activity that are clearly related to the nighttime absence of shortwave radiation. A modeling study of the effects of radiation on tropical cyclones by Hobgood (1986) suggests that the diurnal cycle of net radiation at the cloud tops steepens the lapse rate at night and increases convection. During daylight hours, the absorption of solar radiation reduces the lapse rate, thus reducing convection. This direct radiation-convection interaction may be responsible for the diurnal cycles of convective activity that occur in Experiment 15, and also in the other radiation experiments conducted in this study. A detailed anal-

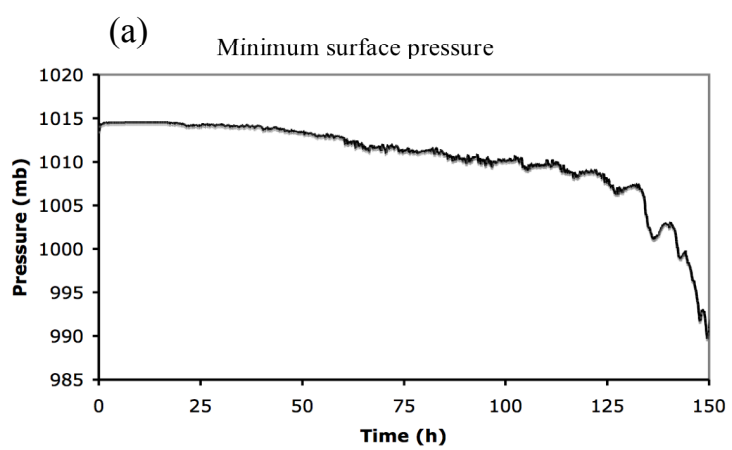

(b)

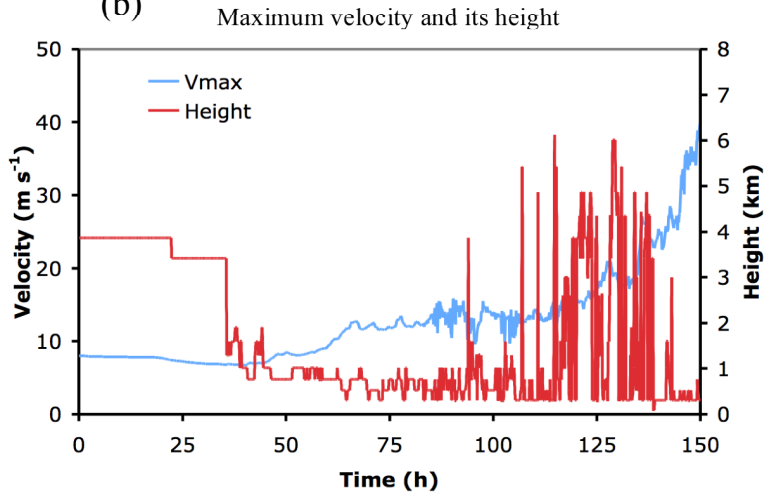

(c) Maximum surface velocity and its radius

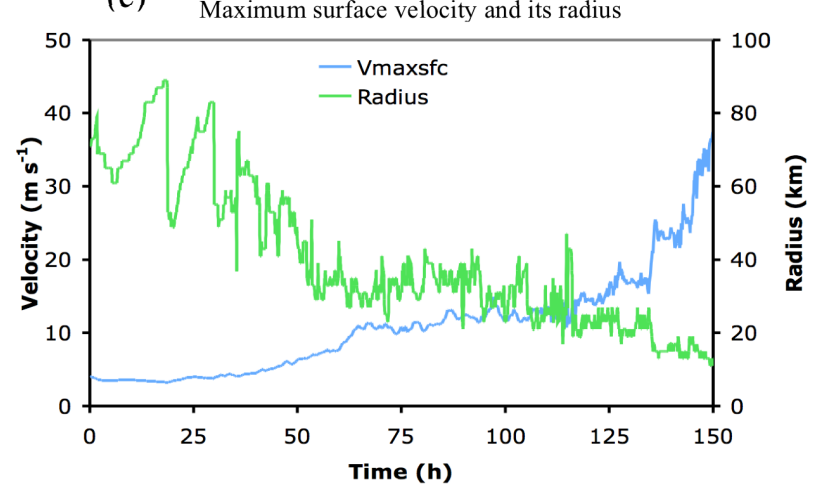

Fig. 10. As in Fig. 2, for Experiment 7.

ysis of the interaction of radiation and convection is beyond the scope of this study, but these results indicate that they are likely to have an important impact on the rate of tropical cyclogenesis and possibly on the pathway taken for this particular posing of the genesis problem within an MCV embryo.

The second mid-level vortex in Experiment 15 develops during the first surge in convective activity at $t=40 \mathrm{~h}$. This second mid-level vortex is short lived relative to the nonradiation case (Fig. 11d and Fig. 9b). A SSCV forms only eight hours after the mid-level vortex develops, at approximately 9 a.m. local time. It then undergoes sustained development through the daytime hours. Although the total liquid and ice in the system core is at a minimum during this period it is still considerably larger than the non-radiation case. 
(a)

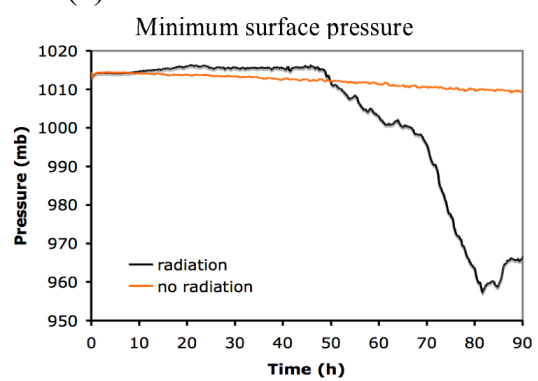

(c)

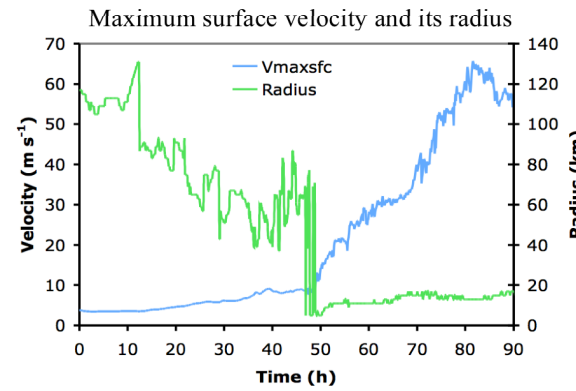

(e)

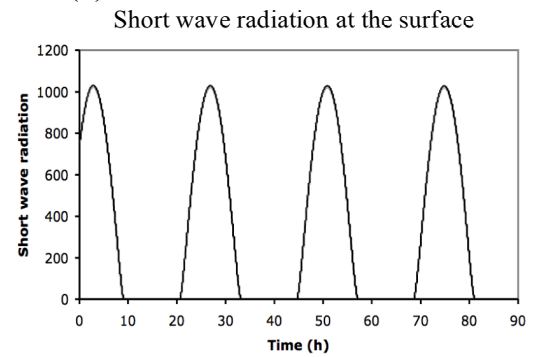

(b)

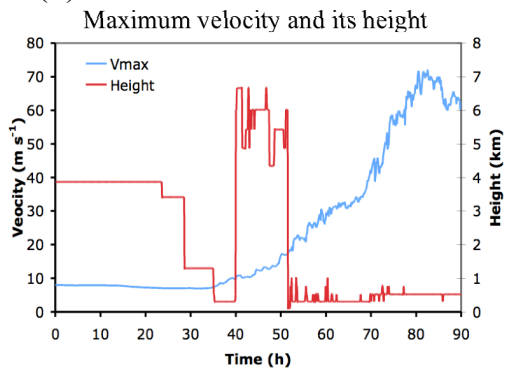

(d)

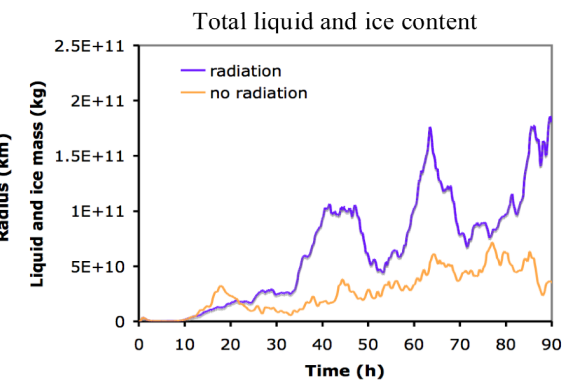

Fig. 11. Time series for Experiment 15. (a) Minimum surface pressure, including comparison with Experiment 6 that is the non-radiation case. (b) The azimuthally averaged maximum tangential velocity and the height it occurs. (c) The azimuthally averaged tangential velocity at the lowest model level and the radius it occurs. (d) Total liquid and ice mass content in a cylindrical volume of radius $100 \mathrm{~km}$ from the center including comparison with the non-radiation case. (e) Clear sky shortwave radiation at the surface.

As the system develops the radius of maximum winds of the small vortex increases from $\sim 5 \mathrm{~km}$ at $t=49 \mathrm{~h}$, to $\sim 17 \mathrm{~km}$ at $t=90 \mathrm{~h}$ (Fig. 11c).

The three radiation simulations that were initialized with a strong initial vortex, Experiments 10, 11, and 13, all developed quickly along pathway One and formed relatively large tropical cyclones. Experiment 10 underwent genesis at approximately $t=30 \mathrm{~h}$. After genesis between $34-45 \mathrm{~h}$, the mid-level winds were generally stronger than at low levels although a well-defined secondary vortex did not form. During this same period the near-surface winds increased significantly from 12 to $20 \mathrm{~m} \mathrm{~s}^{-1}$. Experiment 11, which was identical to Experiment 10 except for the absence of an initial moisture anomaly, underwent genesis at approximately $t=45 \mathrm{~h}$. This case did form a well-defined second mid-level vortex after genesis at $t=47 \mathrm{~h}$. Also, a SSCV formed a few hours later at $t=52 \mathrm{~h}$. This was concurrent with a sudden jump in the radius of maximum winds that decreased to $7 \mathrm{~km}$. However, this small vortex was not persistent and the radius of maximum winds increased to $17 \mathrm{~km}$ three hours later. Therefore, this case was classified as developing via pathway One. Experiment 13 underwent genesis at approximately $t=35 \mathrm{~h}$ evolving along pathway One. Interestingly, a second mid-level vortex developed at $t=39 \mathrm{~h}$ and a SSCV at $t=42 \mathrm{~h}$. This small vortex was weak with only a $2 \mathrm{mb}$ decrease in surface pressure and there was not a concurrent jump in the radius of maximum winds when it formed. It decayed within a couple of hours.

Experiments 14 and 16 are similar to Experiments 5 and 7 , respectively, except that radiation is included. These lower SST cases with radiation also developed along pathway One initially, but weakened subsequently and then underwent genesis a second time along pathway Two. The results of these experiments and Experiment 15 discussed previously 
suggest that inclusion of radiation may make development along pathway Two more likely. However, Experiment 17 that uses the Chen-Cotton radiation scheme, which is identical to Experiment 8 otherwise, showed development along pathway One. The Chen-Cotton scheme is simpler than Harrington's, having less realistic microphysical-radiation interactions. Moreover, comparing the clear sky IR cooling rates showed that they were significantly larger for the ChenCotton scheme than for the Harrington scheme, especially at lower levels. This apparently led to larger values of CAPE developing early on, as well as bringing the air closer to saturation which should favor stronger convective activity during the nighttime between $t=9$ to $21 \mathrm{~h}$. Genesis occurred during this first pulse in convective activity, so that by the early morning the system was developing rapidly and already undergoing intensification. A mid-level vortex did not develop for this case. Although results for the Chen-Cotton scheme are not likely to be as realistic as for the Harrington scheme, this experiment does provide additional evidence that radiation may have significant influence on the pathway taken to genesis.

\subsection{The relation between sea surface temperature and ice production on the pathway taken to genesis}

Comparison of Experiments 1 and 2 clearly demonstrate the importance of the ice phase in promoting development along pathway Two. Furthermore, experiments show that lowering SST when the ice phase is activated can cause genesis to occur along pathway One instead of Two. Development along pathway Two is favored by the formation of a strong mid-level vortex, which is related to a substantial stratiform ice layer aloft and a prominent mid-level inflow. Figure 12 shows the mixing ratio of ice and radial winds for Experiment 2 at $75 \mathrm{~h}$. The ice consists of two layers: A stratiform region above the melting level extending out to $60 \mathrm{~km}$, and a higher anvil layer above $11 \mathrm{~km}$ extending to $140 \mathrm{~km}$. A radial inflow is adjacent to the lower part of the stratiform region with wind speeds of approximately $2 \mathrm{~m} \mathrm{~s}^{-1}$ at a height of $7 \mathrm{~km}$. The inflow has a gradual slope with maximum winds at $140 \mathrm{~km}$ from the center occurring at a height of $8 \mathrm{~km}$. The Coriolis force acting on this inflow air and convergence of the pre-existing circulation appear to be mainly responsible for the spin-up of the mid-level circulation and the strong vortex evident in Fig. 5.

Given the importance of a substantial ice layer for driving a mid-level inflow that produces a strong mid-level vortex, it is a reasonable hypothesis that lower SSTs, which are likely to produce weaker convection and lesser amounts of ice, are more likely to favor development along pathway One. This hypothesis is further supported by comparing the amount of ice and vertical mass transport for Experiment 2, which has an SST of $29^{\circ} \mathrm{C}$ and Experiment 5 that is identical except the SST is $28^{\circ} \mathrm{C}$. Figure $13 \mathrm{a}-\mathrm{c}$ compare the total amount of ice in the fine grid domain, the total amount of ice for radius less
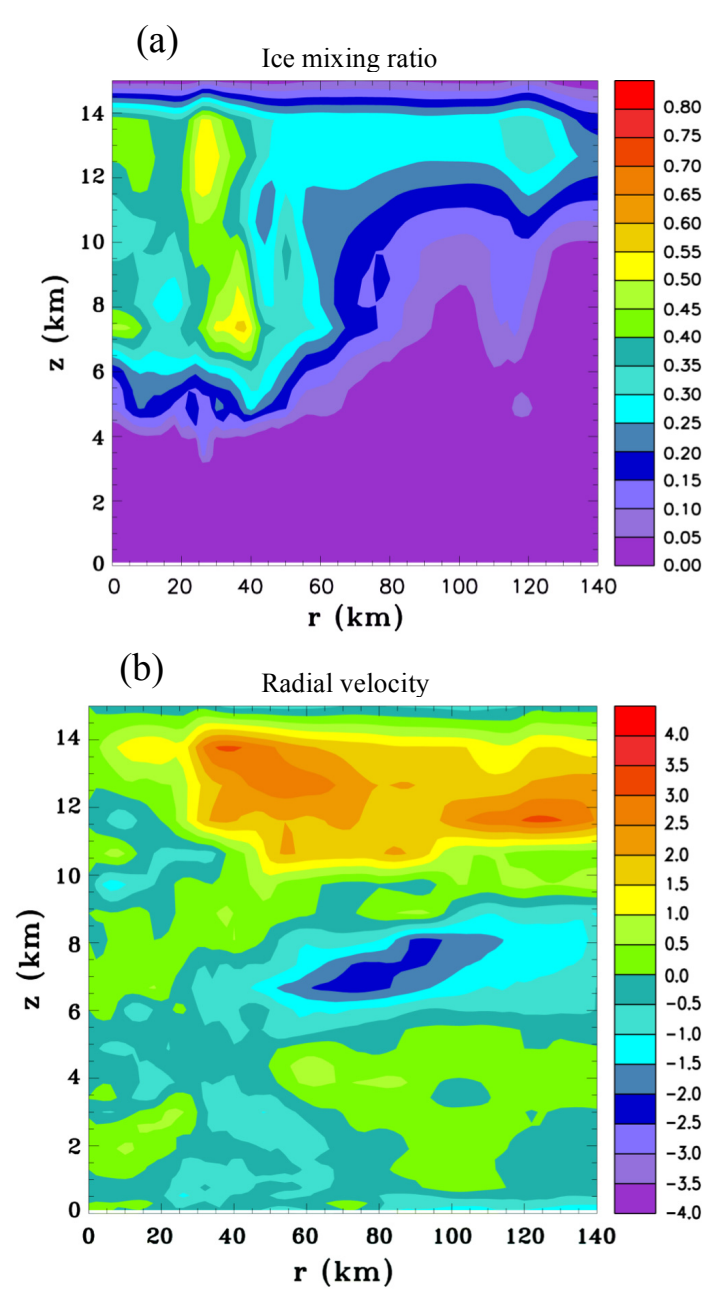

Fig. 12. Azimuthally averaged fields of (a) Mixing ratio of ice (g $\mathrm{kg}^{-1}$ ), and (b) radial component of wind velocity $\left(\mathrm{m} \mathrm{s}^{-1}\right)$, at $t=$ $75 \mathrm{~h}$, for Experiment 2.

than $100 \mathrm{~km}$ and the total vertical mass transport of air at the $7.35 \mathrm{~km}$ level for radius less than $100 \mathrm{~km}$. For Experiment 2, which has a higher SST, there is considerably more ice in the fine grid domain. Also, the total amount of ice occurring in a radius less than $100 \mathrm{~km}$ is more for Experiment 2 although it is not so notable. There are large oscillations evident in the ice content particularly for Experiment 2 that have periods of approximately ten hours. The total vertical mass flux at the $7.35 \mathrm{~km}$ level for radius less than $100 \mathrm{~km}$ also tends to be higher for Experiment 2, particularly early on. Again very large oscillations are evident that are discussed further in Sect. 3.6. These results indicate, as would be expected, that higher SSTs tend to cause stronger convective cells that produce more ice. The outflow layer aloft, seen in Fig. 12b, advects ice away from the center of the system. As the ice falls into drier environmental air at mid-levels, sublimation, melting and evaporation result in cooling that would contribute to driving a mid-level inflow, as will be discussed in Sect. 4. 
(a)

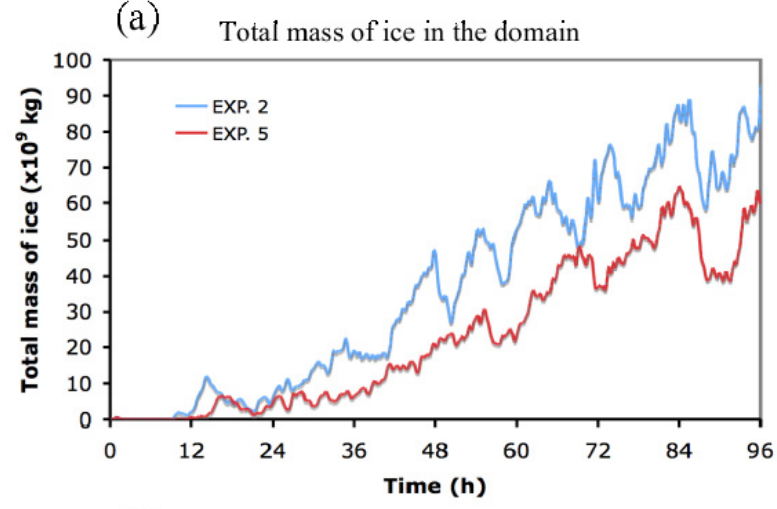

(b)

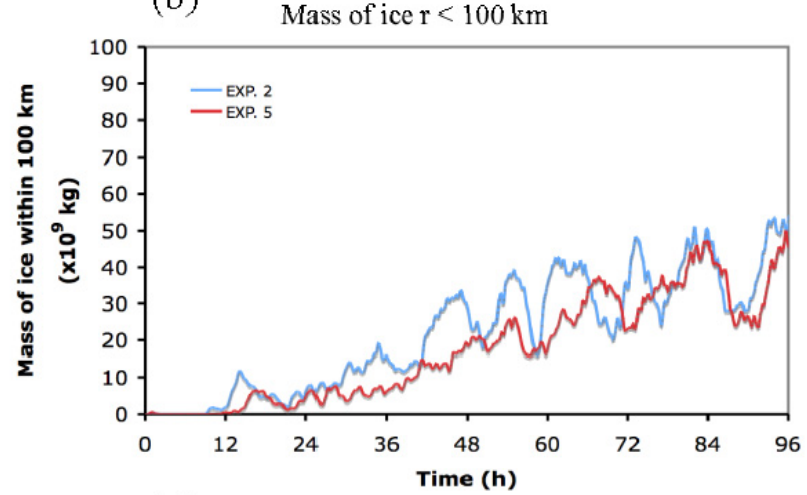

(c) Vertical mass transport $\mathrm{r}<100 \mathrm{~km}, \mathrm{z}=7.35 \mathrm{~km}$

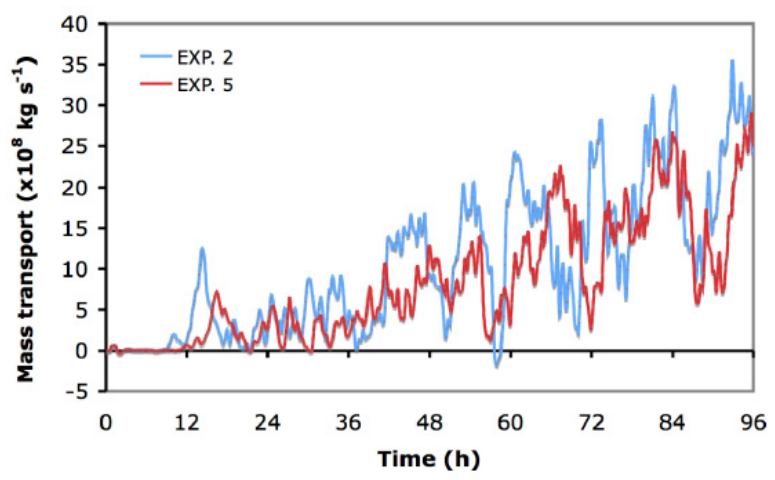

Fig. 13. Comparison of Experiments 2 and 5. (a) The total mass of ice in the fine grid domain. (b) The total mass of ice within a radius of $100 \mathrm{~km}$ of the center of the domain. (c). The vertical mass transport of air within a radius of $100 \mathrm{~km}$ of the center of the domain, at $z=7.35 \mathrm{~km}$.

After a surge in convective activity, a vertical heating profile more similar to the stratiform region of MCSs, which is characterized by latent heating in the upper troposphere and cooling in the lower troposphere (Houze, 1982; Johnson and Young, 1983) is likely to become more prevalent.

\subsection{Formation of the small surface concentrated vortex for cases developing along pathway Two}

Nine of the experiments listed in Table 2 underwent genesis along pathway Two, albeit two of these cases first developed along pathway One before weakening and then strengthening again. In this section we examine the conditions existing prior to the formation of the SSCV and some of the prominent factors that appear important for this process. A detailed diagnosis of each of these simulations that produced the SSCV would be desirable, but is beyond the scope of this exploratory study. This section will focus in some detail on the development of the SSCV for Experiment 2. A brief summary will be given for the other cases with more details provided in Appendix A for the interested reader.

Experiment 2 formed an SSCV at $t=80 \mathrm{~h}$ with winds near the surface reaching $12 \mathrm{~m} \mathrm{~s}^{-1}$ two hours later. At $t=79 \mathrm{~h}$ there was a prominent mid-level vortex (Fig. 5a) and high relative humidity throughout the troposphere in the core of the system. These conditions were identified by N07 as important precursors for the formation of the smaller-scale vortex. Figure 14 shows the azimuthally averaged potential temperature perturbation at $t=79 \mathrm{~h}$. This shows a significant warm anomaly at upper levels in the core of the system. Beneath this warm anomaly there is a cooling between $z=2-4.5 \mathrm{~km}$. It is possible that this cooling, by decreasing the low-level stability, aided the development of convection at the center and the formation of the SSCV. It can also be seen that there is a mean cooling of the air near the surface at the center, which would not be conducive to the development of a convective cell if it was to ingest this air.

The situation preceding the formation of the SSCV was quite complex. Seven hours earlier, at $t=73 \mathrm{~h}$, there was a peak of convective activity that is evident in the time series of ice content and vertical mass flux shown in Fig. 13. At this time there was considerable convective activity southwest of the center that created strong surface outflows and vorticity anomalies. By $t=74 \mathrm{~h}$ a broad region of cold and dry air lay to the south of the center, which was advecting with the cyclonic flow. A strong cell had formed at the eastern edge of this surface outflow $15 \mathrm{~km}$ southeast of the center and had formed a local region of significantly colder and drier air at the surface. Convection continued on the western edge of the broad cold and dry region and at $t=75 \mathrm{~h}$ a strong cell had formed $15 \mathrm{~km}$ due south of the center. There were also active cells $50 \mathrm{~km}$ to the south of the center and cells $30 \mathrm{~km}$ southwest of the center. By this time the very cold air from the earlier southeast cell had advected nearer the center, and the broad region of cool and dry air at the surface was in the southeast quadrant. At $t=76 \mathrm{~h}$ cold downdraft air from the cell that had been $15 \mathrm{~km}$ south of the center was contributing to form a pool of significantly colder and drier air extending from the center to the southeast within the existing broad cold air region. Also, at this time new development had occurred $\sim 5 \mathrm{~km}$ south east of the location of the 


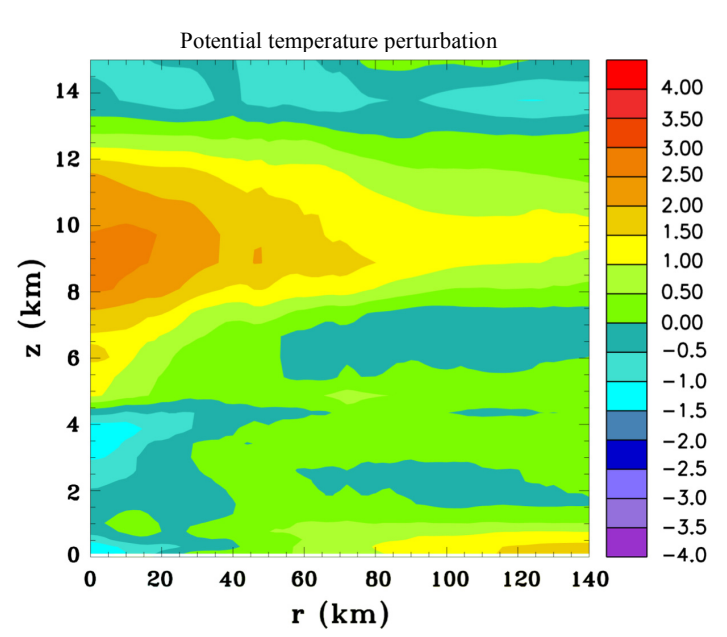

Fig. 14. The azimuthally averaged potential temperature perturbation for Experiment 2, at $t=79 \mathrm{~h}$. The contour interval is $0.5 \mathrm{~K}$.

earlier cell, which was very active and also contributed to forming the cold air anomaly in this region. There was significant low-level positive vorticity in a broad region southwest of the center, a result of the numerous convective cells that had occurred earlier. There was a local surface high pressure anomaly beneath the new active cell, but immediately to the west of it in the center of the low level positive vorticity anomaly a surface mesolow had formed. At $t=77 \mathrm{~h}$, the mesolow had broadened and was trailing the surface cold region, about $20 \mathrm{~km}$ southeast of the center. Convection in this region and throughout the system had declined by this time, as can be seen in Fig. 13. This decline in convective activity was probably primarily due to decreased convective available potential energy (CAPE), as the low-level air was cooled and dried by convective downdrafts. The region of significantly colder air at the surface that was ahead of the low pressure region had moved closer to the center of the system and was merging with the cold air that had previously moved to the center from the earlier southeast cell present at $t=74 \mathrm{~h}$. So the center had become significantly cooler than a few hours previously, and is the main reason for the near-surface cool air anomaly seen in Fig. 14 at $t=79 \mathrm{~h}$.

Figure $15 \mathrm{a}-\mathrm{d}$ show horizontal sections of vertical vorticity, potential temperature, pressure, at $z=98 \mathrm{~m}$, and potential temperature, at $z=7.35 \mathrm{~km}$, for $t=78 \mathrm{~h}$. The vorticity field shows a broad region of mainly positive vorticity anomalies in the core of the system. Numerous vorticity filaments can be identified. They often occur at boundaries between warm and cold air. The relatively cold air is primarily caused by convective-scale downdrafts. The low-level air near the center of the system tends to be colder than the air on the periphery. The mesolow is east of the center and is located on the edge of the surface cold air anomaly. It is a region of enhanced positive vorticity. Also notable is that it
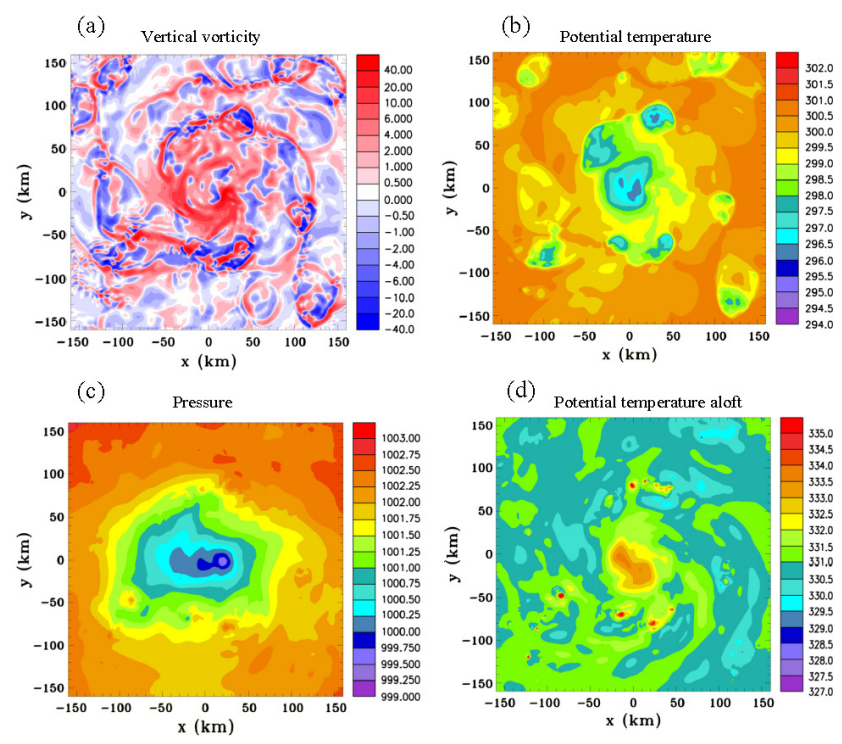

Fig. 15. Horizontal sections for Experiment 2, at $t=78 \mathrm{~h}$. (a) Vertical vorticity $\left(\times 10^{-4} \mathrm{rad} \mathrm{s}^{-1}\right)$, (b) potential temperature $(\mathrm{K}),(\mathbf{c})$ pressure $(\mathrm{mb})$, at $z=98 \mathrm{~m}$, and (d) potential temperature $(\mathrm{K})$, at $z=7.35 \mathrm{~km}$.

is trailed by a long broad filament of positive vorticity that circles around the edge of the central cold pool. Figure 15d shows that the warm core aloft is asymmetrical at this time. The position of the warm air aloft does not correlate well with the mesolow since the latter is a shallow feature. There is a warm air anomaly at low levels within the mesolow of $\sim 1 \mathrm{~K}$ of between $z=1$ to $2 \mathrm{~km}$. At this time the system is still in a state of lowered convective activity as can be seen in Fig. 13. There are a few deep cells evident by the high values of potential temperature aloft in Fig. 15d, at approximately $75 \mathrm{~km}$ from the center, but very little activity in the system core. During the next hour, the mesolow broadened and moved cyclonically to the northeast, approximately $15 \mathrm{~km}$ from the center, remaining on the edge of the coldest air near the center. The quiet period of convective activity had allowed the water vapor mixing ratio near the surface to increase to relatively high values outside the central cold pool. Consequently. convective activity in the system core was starting to become significant again. At this time there was a surface speed maxima approximately $10 \mathrm{~km}$ due north of the center located on the western side of the mesolow, with winds from the north having speeds of $\sim 10 \mathrm{~m} \mathrm{~s}^{-1}$ directed towards the central cold pool. It was at this location on the edge of the central cold pool that the cell developed that formed the SSCV an hour later.

Figure 16a-e show horizontal sections of vertical vorticity, potential temperature, water vapor mixing ratio, pressure - at $z=98 \mathrm{~m}$, and vertical velocity - at $z=1.6 \mathrm{~km}$ for $t=80 \mathrm{~h}$. The SSCV, which has just formed is at the center where there is a very small negative pressure anomaly. It is a region of 

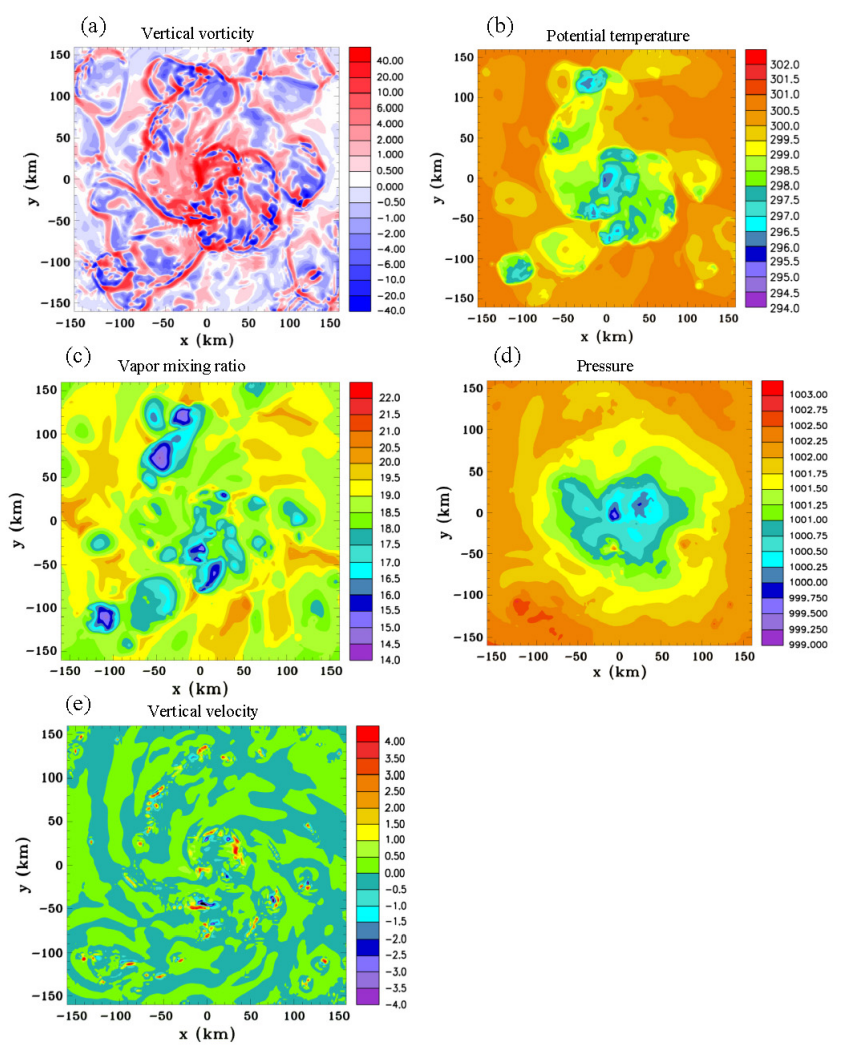

Fig. 16. Horizontal sections for Experiment 2, at $t=80 \mathrm{~h}$. (a) Vertical vorticity $\left(\times 10^{-4} \mathrm{rad} \mathrm{s}^{-1}\right)$, (b) potential temperature $(\mathrm{K}),(\mathbf{c})$ water vapor mixing ratio $\left(\mathrm{g} \mathrm{kg}^{-1}\right)$, (d) pressure $(\mathrm{mb})$, at $z=98 \mathrm{~m}$, and (e) vertical velocity $\left(\mathrm{m} \mathrm{s}^{-1}\right)$, at $z=1.6 \mathrm{~km}$.

strong positive vorticity and there is a moderately strong updraft. The cell ingested relatively warm and moist air from the northwest. The cold pool of air adjacent to the cell is responsible for the near-surface central cold anomaly seen in the azimuthally averaged potential temperature field shown in Fig. 14. Low-level vorticity spiraled into the cell from the northwest during the next hour, which appeared to aid the intensification of the SSCV.

The formation of the SSCV is illustrated in more detail by the zoomed in fields shown in Fig. 17a-f, at $t=79 \mathrm{~h}$. Shown are horizontal sections of CAPE, and pressure, temperature perturbation and vertical velocity at $z=98 \mathrm{~m}$, and vertical sections of potential temperature with contoured $y$ component of velocity $(v)$, and vertical vorticity, at $y=2 \mathrm{~km}$. Wind vectors are also shown in some of the figures. A convective cell has just formed at the center of the domain which is fueled from the north where there are relatively high values of CAPE. There is a circulation around the mesoscale low pressure anomaly and a strong flow from the north impinges on the edge of the coldest air just beneath the updraft base. A strong low-level positive vertical vorticity is slightly northeast of the center which is a source of vorticity for the convective cell. The updraft is located near the center of the
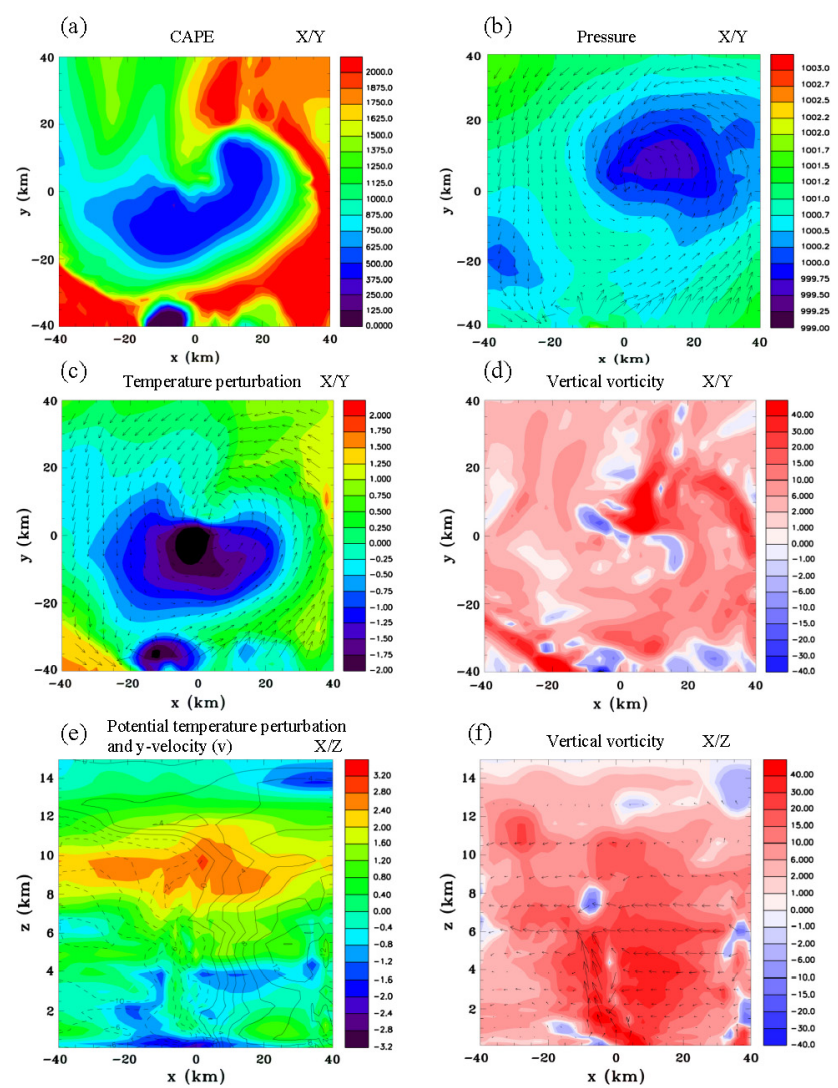

Fig. 17. Zoomed horizontal and vertical sections for Experiment 2, at $t=79 \mathrm{~h}$. (a) CAPE $\left(\mathrm{J} \mathrm{kg}^{-1}\right)$, (b) pressure (mb) and wind vectors $\left(\max 11 \mathrm{~m} \mathrm{~s}^{-1}\right)$, (c) temperature perturbation $(\mathrm{K}),(\mathbf{d})$ vertical vorticity $\left(\times 10-4 \mathrm{rad} \mathrm{s}^{-1}\right)$ and wind vectors, at $z=98 \mathrm{~m}$, and (e) potential temperature perturbation $(\mathrm{K})$ and $y$ velocity $(v)$ contoured at $2 \mathrm{~m} \mathrm{~s}^{-1}$ intervals. (f) Vertical vorticity $\left(\times 10^{-4} \mathrm{rad} \mathrm{s}^{-1}\right)$ and $x-z$ wind vectors $\left(\max 8 \mathrm{~m} \mathrm{~s}^{-1}\right)$, in the $y$ plane at $y=2 \mathrm{~km}$.

mid-level vortex and is relatively warm compared to the surrounding cooler low-level air. The cell at this stage is a developing VHT with high values of positive vorticity. It has weak updraft speeds at this stage of only $6-7 \mathrm{~m} \mathrm{~s}^{-1}$.

Development of the SSCVs for the nine experiments had some elements in common, but there were also some considerable variations in the details. There was usually a preexisting low-level vorticity anomaly that formed $10-25 \mathrm{~km}$ from the center which would subsequently spiral towards the center during the next few hours and either develop into an SSCV, or promote its development. These pre-cursors to an SSCV formed when convection developed in regions of enhanced positive low-level vorticity. This low-level vorticity was itself a product of previous convection, or was currently being generated, for instance by an active surface outflow colliding with a cold pool. The SSCVs all developed very close to the center of the strong mid-level circulation. There was a low-level cold air anomaly between approximately $1.5-5 \mathrm{~km}$, at the center. Also there was a shallow central cold 
pool and the SSCV formed on the edge of it. There were significant positive vertical vorticity anomalies around the central cold pool that appeared to be a significant source of vorticity for the developing SSCV. The SSCV fed on a band of relatively warm and moist air spiraling into the center. The air aloft was close to saturation.

Four of the cases, Experiments 8, 12, 14, and 16, show an interesting and similar pattern of development. A convective cell developed that tilted downstream due to the stronger cyclonic circulation aloft. Rainfall evaporation formed a cold pool downstream of the cell. Upstream of it a shallow small low pressure and positive vertical velocity anomaly developed. This anomaly and the convective cell trailed the cold pool as it spiraled into the center where it intensified into an SSCV.

The SSCV in Experiment 9 developed in a unique way. An MCS that was $50 \mathrm{~km}$ from the domain center created a strong raised mesoscale vortex which played a role in the development of a relatively large SSCV when it reached the center. Another interesting evolution occurred in Experiment 4 where a squall line appeared to have a significant influence on the formation of the SSCV. Further details can be found in Appendix A.

\subsection{Convective-scale downdrafts and oscillations in convective activity}

Surface outflows from convective-scale downdrafts had a major influence on the formation of SSCVs, as discussed in the previous section. The cooling and drying by these downdrafts was typically about $-3 \mathrm{~K}$ and $-3 \mathrm{~g} \mathrm{~kg}^{-1}$, respectively. This is slightly less than observations of boundary layer modification by strong tropical squall lines off the coast of west Africa (Zipser, 1977; Fitzjarrald and Garstang, 1981; Johnson and Nicholls, 1983; Nicholls and Johnson, 1984). One possible reason for this is because prior to genesis there is considerable moistening in the core of the system that would tend to reduce evaporative cooling.

Figure 18 shows time series of the average potential temperature and water vapor mixing ratio within a distance of $100 \mathrm{~km}$ of the center, at $z=305 \mathrm{~m}$, for Experiment 2. Comparing with Fig. 13 that shows the ice content for this case, there is a close correlation especially for the water vapor mixing ratio. For instance, there are minima of the water vapor mixing ratio at $t=47,54,66,75$ and $84 \mathrm{~h}$, and maxima of the ice content at $t=48,56,66,74$, and $86 \mathrm{~h}$. This suggests that as convective downdrafts dry the boundary layer air, convective activity diminishes, leading to less ice production. There is a tendency for the potential temperature anomalies to lag the moisture anomalies. One factor that may contribute to this lag is that the core of the convective downdrafts at this level, are often warmer relative to the ambient air. This is possibly because precipitation drag is playing a significant role in forcing the downdraft in addition to evaporative cooling, so that it is negatively buoyant, in spite of being slightly

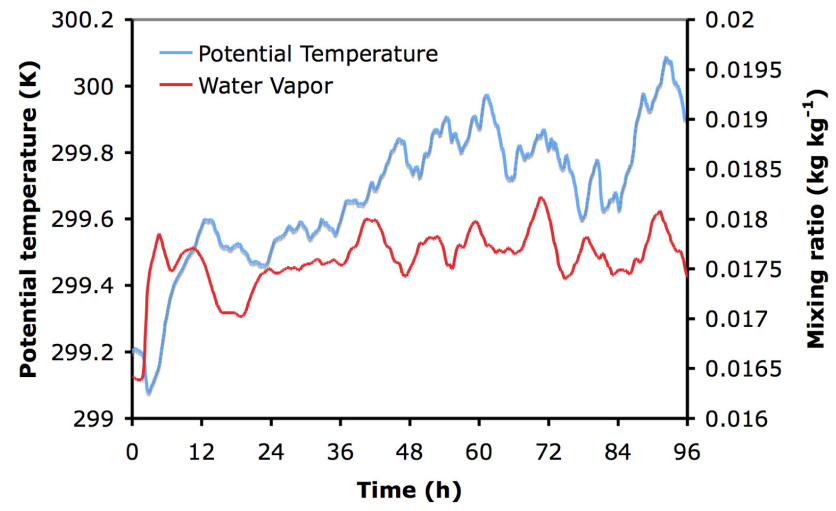

Fig. 18. Time series of the average potential temperature and water vapor mixing ratio within $100 \mathrm{~km}$ of the center, at $z=305 \mathrm{~m}$, for Experiment 2.

warmer than the surrounding air. This was much less notable at the lowest model level, at $z=98 \mathrm{~m}$, where convective downdrafts were almost always cooler than the surrounding air. Another factor is that light rainfall can cause cooling and moistening at low levels, rather than drying. Yet another factor is that compensating subsidence associated with a convectively forced gravity wave can cause warming and drying (Nicholls et al., 1991; Nicholls and Pielke, 2000).

All of the cases without radiation showed significant oscillations in convective activity, although some were much more regular than others. Periods varied, but were typically between $10-15 \mathrm{~h}$. After a period of intense convective activity the boundary layer would gradually recover and convective available potential energy (CAPE) would increase. Convection would often begin fairly close to the center. Spreading surface outflows from this convection would tend to trigger new convection further from the center, and so convective activity would work its way outwards, sometimes as partial rings, or bands of convection, until CAPE was diminished again over a broad area. Most of the cases with radiation activated showed a very clear diurnal cycle of convective activity, as seen in Fig. 11d and e, for Experiment 15. Experiment 17 , on the other hand, only showed one distinct diurnal oscillation at approximately $t=70 \mathrm{~h}$, followed by a steady rise in total condensate. A few of the cases showed small oscillations in between the diurnal cycle. Because of the large amplitude diurnal component in these simulations, the boundary layer recovery is generally prolonged. However, occasionally recovery can take place quickly enough that another oscillation can occur during the daytime.

An SSCV usually formed when convective activity in the system core was increasing, or near a peak. Exceptions are Experiments 4 and 12 that formed an SSCV during a minimum in convective activity. In all the cases simulated, once the SSCV had formed it developed tangential wind speeds of $12 \mathrm{~m} \mathrm{~s}^{-1}$ within $2-3 \mathrm{~h}$. For the cases with radiation activated, 
intensification to tropical storm strength occurred only a few hours later, regardless of the time of day that the SSCV formed. Therefore, development of the SSCV did not appear to be significantly hindered during the daytime by shortwave warming of the upper cloud layer.

\section{Discussion}

For development along pathway Two, the fundamental questions are What principally causes the strong mid-level vortex? Is it important to the formation of the SSCV and why? These questions cannot be definitively answered at this stage. Nevertheless, the experiments that were conducted do shed some light on the processes that are likely to be relevant, in addition to those proposed by N07. Experiments indicate that the ice phase is important for producing a strong mid-level vortex and that a mid-level inflow at the base of the stratiform ice layer precedes its development. The middle level inflow could be explained by axisymmetric balance dynamics associated with the radial derivative of the latent heating due to a "top-heavy" heating profile: latent heating at mid- to upperlevels and cooling at low levels. The response can be considered a slow transverse overturning circulation to the heating distribution. For a $12 \mathrm{~h}$ time average M06 found reasonable agreement for the transverse circulation simulated by RAMS and Eliassen's balanced vortex model (Eliassen, 1951). Alternatively, since the mid-level inflow is likely to be the principal cause of the strong mid-level vortex, and during its development there will be a certain degree of imbalance, another perspective is provided by the simple linear hydrostatic model of thermally forced gravity waves, which neglects the Coriolis force, developed by Nicholls et al. (1991). A solution was obtained for a combined convective and stratiform heating profile, represented by a half-sine wave between the surface and the tropopause and an inverse-sine wave, respectively. The transient response consisted of convective and stratiform forced gravity wave modes, with circulations that laterally expanded away from the heat source region, at a speed depending on the Brunt-Väisäll $\ddot{a}$ frequency and the vertical wave number of the heating. For the slower moving stratiform mode the speed would be approximately $20 \mathrm{~m} \mathrm{~s}^{-1}$ for the tropical atmosphere. This mode is characterized by mid-level inflow and lower- and upper-level outflows. Interestingly, even though the combined heating profile had only a weak net low-level cooling, the stratiform mode that was superimposed with the convective mode still resulted in significant mid-level inflow.

Why the ice phase is important for the development of a mid-level inflow and why cases that produced more ice appear more likely to evolve along pathway Two remains to be answered. The liquid phase of the RAMS model includes small cloud droplets and rain. There is no sedimentation of cloud droplets, whereas rain falls relatively quickly. On the other hand, there is sedimentation of all the ice cate- gories with pristine ice, snow and aggregates generally having slower fall speeds than graupel and hail. With the ice phase included, the anvils of convective towers are composed mainly of ice, rather than cloud droplets, and can fall to lower levels and undergo sublimation and melting. This difference may be a significant factor making it more likely that a stratiform type of heating profile develops. Another possibly important consideration is that the inflow air is relatively dry since it descends from high levels and also because some of it originates from the periphery of the system that has not been moistened so much by vertical convective moisture transport as air at the center. This would tend to increase sublimation and evaporation rates of hydrometeors falling into the inflow, possibly resulting in a positive feedback. Moreover, the inflow advects ice at the base of the stratiform layer towards the center of the system, which would tend to focus cooling near the center, which may aid in the contraction and spin up of the mid-level vortex. Another major difference when ice is included is the additional latent heat of fusion. This could result in enhanced warming aloft and cooling at low levels, increasing the tendency for mid-level convergence.

Additional factors that may influence the spin-up of the mid-level vortex, but are probably of secondary importance to stratiform heating, include: (1) hydrometeor drag that could enhance mid-level convergence when hydrometeors are concentrated at lower levels, and (2) mid-level inflow on the convective scale during the later stage of convective cell development. At this time, latent heating is typically skewed towards upper levels, as melting and evaporation occur at low levels. The development of a convective scale downdraft at low levels with upward motion aloft will induce mid-level convergence.

The SSCV typically formed near to, or at the center of, the strong mid-level vortex. If it formed away from the center it would move cyclonically and spiral into the center of the larger-scale circulation, usually within a few hours. The details of the SSCV formation showed considerable differences. A single intense VHT often played a role in its formation, but sometimes multi-cellular convection was present. For all the cases exhibiting a SSCV, cold pools produced by convective downdrafts played an important role. Vertical vorticity was typically concentrated at the edge of cold pools and new convection that developed in these regions could converge and stretch this pre-existing low-level vorticity. Also, collisions of cold pools could produce convergence and regions of enhanced positive vertical vorticity where they intersected that could favor the formation of an SSCV. A notable factor, usually present, was a significant cold pool at the center of the circulation produced by previous convective activity. An SSCV would often form along its boundary where positive vertical vorticity was concentrated. In a significant number of cases a precursor to a SSCV formed $10-25 \mathrm{~km}$ from the center of the circulation, when a strong convective cell developed above a region of pre-existing low-level vertical vorticity. Because of the stronger cyclonic circulation 
of the mid-level vortex aloft, the updraft became tilted and rain falling on the downstream side of the cell produced a cold pool. A small surface low pressure formed on the upstream side of the cell at the edge of the cold pool, with a prominent positive vertical vorticity anomaly. This could strengthen into an SSCV, or as in the case of Experiment 2, play a role in its formation later on. The small-scale lowpressure anomaly would trail the local cold pool, remaining on its edge as it spiraled in towards the center. Intensification of convection would often occur on the edge of the pressure low as it neared the center and this may have been facilitated by the cold air at mid-to-low levels at the center of the midlevel vortex, which would tend to decrease low-level static stability. The convection fed on a band of relatively moist and warm air spiraling towards the center and because of the reduced low-level static stability at the center would probably be in a region of increased low-level CAPE. The SSCV would then in most cases develop rapidly into a tropical cyclone. The clearest examples of this behavior occurred in Experiments 8,12, 14 and 16, discussed in Sect. 3.5. Therefore, it appears that the formation of a prominent mid-level vortex, in addition to causing a large increase in the efficiency of the conversion of latent heat energy to the kinetic energy of the cyclonic wind field as found by N07, may play a role in favoring the development of an SSCV due to its vertical wind shear and decreased low-level static stability at its center.

There was a fortuitous aspect to the formation and intensification of an SSCV. Whether a small surface pressure anomaly would survive or intensify as it spiraled towards the center depended on a supply of relatively warm and moist low-level air on its outer flank to fuel convection. This supply could be disrupted by downdraft outflow of cold and dry air from nearby convection. There were however cases where surface outflow from nearby convection appeared to enhance low-level convergence and positive vertical vorticity in the vicinity of the small pressure anomaly that promoted the development of an SSCV (e.g. Experiments 4, 9 and 12 discussed in Sect. 3.5).

A complementary interpretation as to why the simulated evolution does not continue to evolve along pathway One is that as the strong, second mid-level vortex develops, the lowlevel cooling in the system core would produce a divergent tendency at low levels that might arrest the contraction of the surface wind speed maxima. The foregoing thermodynamic and dynamic interpretations need not be mutually exclusive and future work should quantify their relative importance.

Inclusion of active radiation is found to significantly increase the rate of genesis. This appears to be largely explained by clear sky cooling due to long-wave radiation at upper levels causing CAPE to increase as well as bringing the air closer to saturation. The Harrington radiation scheme slightly favored evolution along pathway Two, probably because of cooling aloft promoting ice production; whereas, the Chen-Cotton scheme favored evolution along pathway One, apparently due to stronger long-wave cooling, partic- ularly at lower levels, causing the early convective activity to be intense enough to quickly spin up the low-level winds to tropical cyclone strength. Additionally, a strong diurnal oscillation of convective activity was present that is probably mainly due to daytime warming of the upper cloud layer causing an increase in stability aloft, as found by Hobgood (1986). However, even in the complete absence of radiation, large amplitude convective oscillations usually occurred with a shorter period, typically between $10-15 \mathrm{~h}$, that were associated with boundary layer modification by convective downdrafts. When radiation was included a smaller surge in convective activity sometimes occurred in the daytime, between diurnal peaks. This indicates that the boundary layer recovery was often significantly shorter than the diurnal cycle. Both diurnal and semi-diurnal cycles have been found in the areal extent of the upper level cirrus deck of fully developed tropical cyclones (Browner et al., 1977; Muramatsu, 1983; Lajoie and Butterworth, 1984; Steranka et al., 1984; Kossin, 2002). The results presented here for the early stage of a developing system may be relevant to these previous studies, although the existence of substantial convective downdrafts and cold pools in the core of developing tropical cyclones has not yet been established definitively.

\section{Conclusions}

Results of idealized numerical simulations suggest that there are two canonical pathways that can lead to tropical cyclone genesis from an initial weak mid-level vortex. One pathway proceeds by spin up of surface winds that remain stronger than, or of comparable magnitude to, winds aloft until they become large enough that they reach tropical depression strength, defined herein as wind speeds exceeding $12 \mathrm{~m} \mathrm{~s}^{-1}$. Vorticity is concentrated in numerous short-lived rotating deep convective towers that collectively drive a system scale inflow at low levels. Vorticity gradually builds in the central region as the system scale inflow produces increasing cyclonic vorticity and as small-scale cyclonic vorticity anomalies generated by the VHTs are converged at low levels and aggregate. This is usually a stochastic process with no prominent events leading to sudden falls of the surface pressure. The characteristics of pathway One are consistent with the stochastic view of genesis as articulated by Ooyama (1982).

The second pathway at early times proceeds in the same manner as the first, but before surface winds reach tropical cyclone strength the mid-level winds increase significantly and become stronger than the near-surface tangential winds. The low-level air becomes relatively cold (between approximately $1.5-5 \mathrm{~km}$ ) in the core as the second stronger midlevel vortex develops. This is then followed by the sudden formation of a smaller-scale vortex at the center of the largerscale circulation. The small vortex is warm core and produces a narrow column of warm air within the relatively 
colder low-level air associated with the mid-level vortex. It extends upwards into the larger-scale warm core aloft. Its rapid formation often leads to a sudden local decrease of the minimum surface pressure of a few millibars. Once formed it is resilient and becomes the focus of a strengthening and widening vorticity center. Sometimes the pressure continues to fall immediately following this event and the system intensifies rapidly. Evolution along pathway Two has similarities to the conceptual model of Bister and Emanuel (1997; Fig. 13, Sect. 8) that places emphasis on the role of the midlevel vortex. However, the development of low-level vorticity in these simulations does not appear to be primarily due to a top down process, as they envision. The surface wind speeds of some of the simulated systems evolving along pathway Two approach or reach tropical cyclone strength prior to a prominent mid-level vortex developing so that the subsequent formation of a SSCV in these cases could be considered intensification rather than genesis.

Systems that evolved along pathway Two developed a second mid-level vortex that was considerably stronger than typically occurs in the stratiform region of an MCS, with wind speeds in excess of $12 \mathrm{~m} \mathrm{~s}^{-1}$. For the experiments conducted, its horizontal scale as measured by the radius of maximum tangential winds ranged from 23 to $91 \mathrm{~km}$, at the time the SSCV formed. It was shown that the ice phase played an important role in producing a strong second mid-level vortex. Mid-level inflow that developed at the base of the ice layer appeared to be the main factor responsible for its formation. Results suggest that higher SSTs lead to more ice production, which favors the development of a strong mid-level vortex and evolution along pathway Two.

There were significant differences in the way that the SSCV formed for cases that developed along pathway Two. In all cases there appeared to be significant stretching of pre-existing low-level vertical vorticity anomalies by convective scale updrafts. Cold pools produced by convective scale downdrafts played important roles in the formation process. In some cases, the vertical wind shear associated with the strong mid-level vortex influenced the convective activity and development of a small low-level vorticity anomaly that would later either evolve into the SSCV, or play a part in its formation.

Implementation of active radiation in the model via the Harrington scheme increased the tendency to develop along pathway Two. In contrast, the Chen-Cotton radiation scheme increased the tendency to develop along pathway One. Both schemes led to an increase in the rate of genesis compared to non-radiation cases that were otherwise identical. Strong diurnal oscillations in convective activity occurred with radiation activated. SSCVs that formed during the daytime when net convective activity was reduced did not appear to be significantly hindered from developing.

The RAMS results indicate that this initial value problem may be near a bifurcation point, so that changes to model physics could have considerable impact on the outcome of experiments and the likelihood of development along a particular pathway. There are outstanding questions regarding the physical processes, involved in the formation of the strong mid-level vortex and the SSCV, that occur during development along pathway Two. In particular, the role of the mid-level vortex in the sudden formation of an SSCV needs to be further understood. At present, there is no conclusive observational evidence of a tropical cyclone undergoing genesis along pathway Two, and so for the time being the existence of this pathway remains hypothetical, being based on the results of numerical modeling studies with WRF (N07) and with RAMS in this study. The uncertainty in the realism of the second pathway simulated in these idealized configurations and in N07 offer some motivation to examine recent observational data collected during the Tropical Cyclone Structure-2008 (TCS-08) and the Pre-Depression Investigation of Cloud Systems in the Tropics (PREDICT) experiments to examine if some TCs do indeed develop in the manner of pathway Two.

\section{Appendix A}

\section{Further details of the formation of the SSCV for specific experiments}

For Experiment 3, the origins of the SSCV could be traced back to the surface outflow from a strong convective cell centered $70 \mathrm{~km}$ to the east of the center, which collided with a pool of cold air just to the east of the center. This caused strong easterlies just to the east of the center, whereas just south of this were the southwesterlies of the cyclonic circulation. A VHT with a weak surface low formed in this region of positive low-level vertical vorticity, $15 \mathrm{~km}$ east of the center. It remained on the edge of the more central cold pool circling once around the center and within three hours had become an SSCV that was intensifying rapidly. By $t=151 \mathrm{~h}$ tangential wind speeds of the SSCV had reached $12 \mathrm{~m} \mathrm{~s}^{-1}$, but a cluster of cells to the north produced a strong surface outflow that shortly afterwards swept across the center temporarily weakening the SSCV, as can be seen in the minimum surface pressure time series shown in Fig. 6.

For Experiment 4, a cluster of cells approximately $20 \mathrm{~km}$ northwest of the center produced a surface cold pool, and a new cell formed on its southern edge where there was significant positive vertical vorticity. This VHT had a small surface low and during the next two hours moved cyclonically to $15 \mathrm{~km}$ southeast of the center intensifying into an SSCV. During this period a squall line formed west of the center that rapidly propagated southwards. The squall line did not impede the development of the SSCV, which fed on a narrow band of warm and moist air that was squeezed between the older cold pool to the north and the squall line surface outflow to the south. It is possible that the strong southwesterly 
surface winds just south of the cell caused by the squall line outflow may have contributed to the vorticty of the SSCV.

For Experiment 8, a strong isolated cell $20 \mathrm{~km}$ from the center created a cold pool of air at the surface. The cyclonic winds that were stronger aloft due to the strong mid-level vortex tilted the cell down shear, so that the rain shaft and low-level downdraft was downstream of the updraft. At this time the surface outflow winds were strong and the cold and dry air spread out at the surface downstream of the cell. A small and weak surface low formed just upstream of the rain shaft and it was a region of notably stronger positive vertical vorticity. The cell trailed the cold pool around the center for the next three hours feeding on low-level air from its right rear quadrant. This air de-accelerated after entering the lowpressure region and fed the updraft that was just downstream of the low. As the cold pool moved closer to the center the cell moved cyclonically around it and intensified to form an SSCV $10 \mathrm{~km}$ from the center.

For Experiment 9, the evolution was significantly different than the other experiments with a relatively large SSCV forming having a radius of $11 \mathrm{~km}$. A short-lived MCS formed $50 \mathrm{~km}$ from the domain center. In its decaying stage a raised mesoscale low pressure anomaly with a cyclonic circulation developed between $z=2-6 \mathrm{~km}$. The raised mesoscale vortex was a persistent feature that moved cyclonically and spiraled towards the center in the next few hours. At this point a large convective cluster explosively developed $30 \mathrm{~km}$ from the center and formed a strong surface outflow that interacted with convection within the mesoscale vortex. Two hours later, a surface low had formed at the center. Transient multicellular convection occurred around the edge of the surface mesolow, rather than near its center as the SSCV intensified. It is possible that because there was a significant circulation present between $z=2-4 \mathrm{~km}$ when the SSCV developed that convective downdrafts may have brought some of the cyclonic vorticity down to the surface. There were however significant pockets of low-level positive vertical vorticity near the surface prior to the SSCV forming, as well as the convective surface outflows that appear to favor its development, so it probably formed mainly from the bottom up.

For Experiment 12 the mid-level vortex was very strong prior to the SSCV forming with maximum winds of $19.1 \mathrm{~m} \mathrm{~s}^{-1}$ and a large radius of $71 \mathrm{~km}$. The origins of the SSCV could be traced back to a small weak surface low with a positive vertical vorticity anomaly $25 \mathrm{~km}$ from the center. The low was upstream of a local cold pool and a large convective cell. Rainfall from the convective cell was producing the cold pool. During the next two hours the small low moved cyclonically round the center trailing the cold pool showing similarities to Experiment 8. At $t=53 \mathrm{~h}$ it was $15 \mathrm{~km}$ east of the center. A strong convective cell that had developed $65 \mathrm{~km}$ to the northeast of the center produced a strong outflow that enhanced the easterly flow to the north of the small low. At $t=54 \mathrm{~h}$, the pressure in the small low had fallen significantly and it had become an SSCV $25 \mathrm{~km}$ north of the center. There was also significant positive vorticity anomalies on the surface outflow boundary of the cold pool produced by the cell to the northeast that appeared to be merging into the SSCV, and may have contributed to its development.

For Experiment 14 genesis first occurred along pathway One at $t=37 \mathrm{~h}$, followed by weakening, and then redevelopment along pathway Two, forming an SSCV at $t=66 \mathrm{~h}$. At $t=57 \mathrm{~h}$ the cyclonic circulation at the surface was still quite strong with speeds of $\sim 7 \mathrm{~m} \mathrm{~s}^{-1}$ between a radius of $25-70 \mathrm{~km}$ from the center. A mid-level vortex was present with winds of $12 \mathrm{~m} \mathrm{~s}^{-1}$ at a radius of $35 \mathrm{~km}$ and a height of $6 \mathrm{~km}$. The central air at the surface was not very cold at this time and the water vapor mixing ratio was high. This promoted convective activity during the next few hours that produced convergence and spin up of the low-level winds and a broad region of cold surface air at the center of the system. Also, a considerable amount of ice was produced aloft and a notable mid-level inflow developed. The net result was that at $t=63 \mathrm{~h}$, there was a considerably stronger surface circulation with maximum mean wind speeds of $\sim 9 \mathrm{~m} \mathrm{~s}^{-1}$ at a radius of $15 \mathrm{~km}$ and a smaller more intense mid-level vortex aloft. At $t=65 \mathrm{~h}$, a strong convective cell developed $10 \mathrm{~km}$ from the center and a downdraft formed downstream producing a local cold pool trailed by a small surface pressure low, similarly to Experiments 8 and 12. This small scale vorticity anomaly intensified into an SSCV just inside the wind speed maxima of the larger-scale circulation. During the next few hours, the SSCV spiraled towards the center and intense convection within it converged the relatively strong low-level circulation to form a small and intense vorticity center.

For Experiment 15, a second mid-level vortex of only moderate size developed, with a radius of $47 \mathrm{~km}$, even though the initial vortex was large. This appeared to be because convection tended to be more focused in the core of the system because the lower SST of $28^{\circ} \mathrm{C}$ led to a reduction of outer convection. At $t=47 \mathrm{~h}$ a strong cell developed $10 \mathrm{~km}$ north of the center and its downdraft significantly cooled the central region. At $t=48 \mathrm{~h}$ a surface outflow from a very active cluster $50 \mathrm{~km}$ to the northeast produced strong easterlies at approximately $25 \mathrm{~km}$ north of the center that collided with the central cold pool. At this intersection, where there was strong surface horizontal wind shear a cell formed that quickly developed a small and intense low-level positive vertical vorticity anomaly. By $t=49 \mathrm{~h}$, the SSCV was located on the western edge of the central cold pool and developing rapidly.

For Experiment 16, a small mid-level vortex formed with a radius of only $23 \mathrm{~km}$. Surface winds were fairly strong, near the center of the system when the mid-level vortex formed because it had previously undergone genesis along pathway One, similarly to Experiment 14 . At $t=66 \mathrm{~h}$ there was a significant cold pool at the center produced by recent convective activity in this region. Surface winds were relatively light within the cold pool, but adjacent to its edge at a radius of $30 \mathrm{~km}$ there was a significant cyclonic circulation with wind 
speeds of $\sim 9 \mathrm{~m} \mathrm{~s}^{-1}$. Convection became more active within the radius of maximum winds with one of the cells forming a significant small surface low $15 \mathrm{~km}$ from the center. The cell moved cyclonically trailed by the small low similarly to Experiments 8, 12 and 14. It intensified into an SSCV on the edge of the central cold pool. At this stage its surface winds were considerably stronger than the larger-scale circulation that could still be identified at a radius of $\sim 30 \mathrm{~km}$.

Acknowledgements. We are grateful to Saurabh Barve for providing computational assistance, and Thomas Cram, Wesley Terwey and Donavan Wheeler for aid with data analysis code. This work was supported in part by the National Science Foundation, under Grants NSF AGS 0965721 and ATM-0733380, and by the U.S. Office of Naval Research Grant No. N00014-03-1-0185.

Edited by: T. J. Dunkerton

\section{References}

Bister, M. and Emanuel, K. A.: The genesis of hurricane Guillermo: TEXMEX analyses and a modeling study, Mon. Weather Rev., 125, 2662-2682, 1997.

Braun, S. A.: A cloud-resolving simulation of Hurricane Bob (1991): Storm structure and eyewall buoyancy, Mon. Weather Rev., 130, 1573-1592, 2002.

Braun, S. A., Montgomery, M. T., Mallen, K. J., and Reasor, P. D.: Simulation and interpretation of the genesis of tropical storm GERT(2005) as part of the NASA tropical cloud systems and processes experiment, J. Atmos. Sci., 67, 999-1025, 2010.

Browner, S. P., Woodley, W. L., and Griffith, C. G.: Diurnal oscillation of the area of cloudiness associated with tropical storms, Mon. Weather Rev., 105, 856-864, 1977.

Charnock, H.: Wind stress on a water surface, Q. J. Roy. Meteorol. Soc., 81, 639-640, 1955.

Chen C. and Cotton, W. R.: The physics of the marine stratocumulus-capped mixed layer,, J. Atmos. Sci., 44, 2951-2977, 1987.

Clark, T. L. and Farley, R. D.: Severe downslope windstorm calculations in two and three spatial dimensions using anelastic grid nesting: A possible mechanism for gustiness, J. Atmos. Sci., 41, 329-350, 1984.

Davis, C. A. and Bosart, L. F.: Numerical simulations of the genesis of Hurricane Diana (1984). Part 1: Control Simulation, Mon. Weather Rev., 129, 1859-1881, 2001.

Dunkerton, T. J., Montgomery, M. T., and Wang, Z.: Tropical cyclogenesis in a tropical wave critical layer: easterly waves, Atmos. Chem. Phys., 9, 5587-5646, doi:10.5194/acp-9-5587-2009, 2009.

Eliassen, A.: Slow thermally or frictionally controlled neridional circulation in a circular vortex, Astrophys. Norv., 5, 19-60, 1951.

Fitzjarrald, D. R. and Garstang, M.: Vertical structure of the tropical boundary layer, Mon. Weather Rev., 109, 1512-1526, 1981.

Gray, W. M. and Jacobson, R. W. J.: Diurnal variation of deep cumulus convection, Mon. Weather Rev., 105, 1171-1188, 1977.
Hack, J. J. and Schubert, W. H.: Nonlinear response of atmospheric vortices to heating by organized cumulus convection, J. Atmos. Sci., 43, 1559-1573, 1986.

Harr, P. A. and Elsberry, R. L.: structure of a mesoscale convective system embedded in Typhoon Robya during TCM-93, Mon. Weather Rev., 124, 634-652, 1996.

Harr, P. A., Kalafsky, M. S., and Elsberry, R. L.: Environmental conditions prior to formation of a midget tropical cyclone during TCM-93, Mon. Weather Rev., 124, 1693-1710, 1996.

Harrington, J. Y., Feingold, G., and Cotton, W. R.: Radiative impacts on the growth of a population of drops within summertime arctic stratus, J. Atmos. Sci., 57, 766-785, 2000.

Hendricks E. A., Montgomery, M. T., and Davis, C. A.: On the role of "vortical" hot towers on tropical cyclone formation, J. Atmos. Sci., 61, 1200-1232, 2004.

Hill, G. E.: Factors controlling the size and spacing of cumulus clouds as revealed by numerical experiments, J. Atmos. Sci., 31, 646-673, 1974.

Hobgood, J. S.: A possible mechanism for the diurnal oscillation of tropical cyclones, J. Atmos. Sci., 43, 2901-2922, 1986.

Houze Jr., R. A.: Cloud clusters and large-scale vertical motions in the tropics, J. Meteor. Soc. Jpn., 60, 396-410, 1982.

Johnson, R. H. and Nicholls, M. E.: A composite analysis of the boundary layer accompanying a tropical squall line, Mon. Weather Rev., 111, 308-319, 1983.

Johnson, R. H. and Young, G. S.: Heat and moisture budgets of tropical mesoscale anvil clouds, J. Atmos. Sci., 40, 2138-2147, 1983.

Jordan, C. L.: Mean soundings for the West Indies area, J. Meteor., 15, 91-97, 1958.

Klemp, J. B. and Wilhelmson, R. B.: The simulation of threedimensional convective storm dynamics, J. Atmos. Sci., 35, 1070-1086, 1978.

Kossin, J. P.: Daily hurricane variability inferred from GOES infrared imagery, Mon. Weather Rev., 130, 2260-2270, 2002.

Lajoie, F. A. and Butterworth, I. J.: Oscillation of high-level cirrus and heavy precipitation around Australian region tropical cyclones, Mon. Weather Rev., 112, 535-544, 1984.

Lilly, D. K.: On the numerical simulation of buoyant convection. Tellus, 14, 148-172, 1962.

Liu, Y., Zhang, D.-L., and Yau, M. K.: A multiscale numerical study of Hurricane Andrew (1992). Part 1: Explicit simulation and verification, Mon. Weather Rev., 125, 3073-3093, 1997.

Louis, J.-F.: A parametric model of vertical eddy fluxes in the atmosphere, Bound.-Lay. Meteor., 17, 187-202, 1979.

Mapes, B. E. and Houze, R. A.: Diabatic divergence profiles in western Pacific mesoscale convective systems, J. Atmos. Sci., 52, 1807-1828, 1995.

Montgomery M. T. and Smith, R. K.: Tropical cyclone formation: Theory and idealized modeling: Report for the Seventh International Workshop on Tropical Cyclones, La Reunion, Nov. 2010. World Meteorological Organization, Geneva, Switzerland, 2010.

Montgomery, M. T. and Smith, R. K.: The genesis of Typhoon Nuri as observed during the Tropical Cyclone Structure 2008 (TCS08) field experiment - Part 2: Observations of the convective environment, Atmos. Chem. Phys., 12, 4001-4009, doi:10.5194/acp-124001-2012, 2012.

Montgomery, M. T., Nicholls, M. E., Cram, T. A., and Saunders, A. B.: A vortical hot tower route to tropical cyclogenesis, J. Atmos. 
Sci., 63, 355-386, 2006.

Montgomery, M. T., Wang, Z., and Dunkerton, T. J.: Coarse, intermediate and high resolution numerical simulations of the transition of a tropical wave critical layer to a tropical storm, Atmos. Chem. Phys., 10, 10803-10827, doi:10.5194/acp-1010803-2010, 2010.

Montgomery, M. T., Davis, C., Dunkerton, T., Wang, Z., Velden, C., Torn, R., Majumdar, S. J., Zhang, F., Smith, R. K., Bosart, L., Bell, M. M., Haase, J. S., Heymsfield, A., Jensen, J., Campos, T., and Boothe, M. A.: The Pre-Depression Investigation of Cloud Systems in the Tropics (PREDICT) Experiment: Scientific Basis, New Analysis Tools, and Some First Results, Bu. Am. Meteor. Soc., 93, 153-172, 2012.

Muramatsu, T.: Diurnal variations of satellite-measured $T_{B B}$ areal distribution and eye diameter of mature typhoons, J. Meteor. Soc. Japan, 61, 77-89, 1983.

Nicholls, M. E. and Johnson, R. H.: A model of a tropical squall line boundary layer wake, J. Atmos. Sci., 41, 2774-2792, 1984.

Nicholls, M. E. and Pielke, R. A.: Thermally-induced compression waves and gravity waves generated by convective storms, J. Atmos. Sci., 57, 3251-3271, 2000.

Nicholls, M. E., Pielke, R. A., and Cotton, W. R.: Thermally forced gravity waves in an atmosphere at rest, J. Atmos. Sci., 48, 18691884, 1991.

Nolan, D. S.: What is the trigger for tropical cyclogenesis?, Aust. Met. Mag., 56, 241-266, 2007.

Ooyama K.: Numerical simulation of the life cycle of tropical cyclones, J. Atmos. Sci., 26, 3-40, 1969.

Randall, D. A., Harshvardhan, and Dazlich, D. A.: Diurnal variability of the hydrologic cycle in a general circulation model, J. Atmos. Sci., 48, 40-62, 1991.

Rappin, E. D., Nolan, D. S., and Emanuel, K. A.: Thermodynamic control of tropical cyclogenesis in environments of radiativeconvective equilibrium with shear, Q. J. Roy. Meteor. Soc., 136, 1954-1971, 2010.

Raymond, D. J. and López Carrillo, C.: The vorticity budget of developing typhoon Nuri (2008), Atmos. Chem. Phys., 11, 147163, doi:10.5194/acp-11-147-2011, 2011.

Raymond, D. J., Lopez Carrillo, C., and Lopez Cavazos, I.: Case studies of developing east Pacific easterly waves, Q. J. Roy. Meteor. Soc., 124, 2005-2034, 1998.

Raymond, D. J., Sessions, S. L., and López Carrillo, C.. Thermodynamics of tropical cyclogenesis in the northwest Pacific, J. Geophys. Res., D18101, 18, 2011 doi:10.1029/2011JD015624, 2011.

Reasor, P., Montgomery, M. T., and Bosart, L.: Mesoscale observations of the genesis of hurricane Dolly (1996), J. Atmos. Sci., 62, 3151-3171, 2005.

Ritchie, E. A. and Holland, G. J.: Scale interactions during the formation of Typhoon Irving, Mon. Weather Rev., 125, 1377-1396, 1997.
Rogers, R. F., Chen, S. S., Tenerelli, J. E., and Willoughby, H. E.: A numerical study of the impact of vertical shear on the distribution of rainfall in Hurricane Bonnie (1998), Mon. Weather Rev., 131, 1577-1599, 2003.

Schubert, W. H., Hack, J. J., Silva Dias, P. L., and Fulton, S. R.: Geostrophic adjustment in an axisymmetric vortex, J. Atmos. Sci., 37, 1464-1484, 1980.

Simpson, J., Ritchie, E., Holland, G. J., Halverson, J., and Stewart, S.: Mesoscale interactions in tropical cyclogenesis, Mon. Weather Rev., 125, 2643-2661, 1997.

Sippel, J. A., Nielsen-Gammon. J. W., and Allen, S. E.: The multiple-vortex nature of tropical cyclogenesis, Mon. Weather Rev., 134, 1796-1814, 2006.

Smagorinsky, J. S.: General circulation experiments with the primitive equations. 1: The basic experiment, Mon. Weather Rev., 91, 99-164, 1963.

Smith, R. K. and Montgomery, M. T.: Observations of the convective environment in developing and non-developing tropical disturbances, Q. J. Roy. Meteorol. Soc., 138, 1721-1739, 2012.

Steranka, J., Rodgers, E. B., and Gentry, R. C.: The diurnal variation of Atlantic ocean tropical cyclone cloud distribution inferred from geostationary satellite infrared measurements, Mon. Weather Rev., 112, 2338-2344, 1984.

Tripoli, G. J. and Cotton, W. R.: The use of ice-liquid water potential temperature as a thermodynamic variable in deep atmospheric models, Mon. Weather Rev., 109, 1094-1102, 1981.

Walko, R. L., Cotton, W. R., Harrington, J. L., and Meyers, M. P.: New RAMS cloud microphysics parameterization. Part I: The single-moment scheme, Atmos. Res., 38, 29-62, 1995.

Wang, Z., Montgomery, M. T., and Dunkerton, T. J.: Genesis of Pre-hurricane Felix (2007). Part 1: The role of the easterly wave critical layer, J. Atmos. Sci., 67, 1711-1729, 2010.

Zehr, R. M.: Tropical cyclogenesis in the western North Pacific. NOAA Tech. Rep. NESDIS 61, 181 pp., Available from U.S. Department of Commerce, NOAA/NESDIS, 5200 Auth Rd., Washington, DC 20233, 1992.

Zhang, C. and Chou, M.-D.: Variability of water vapor, infrared radiative cooling, and atmospheric instability for deep convection in the equatorial western Pacific, J. Atmos. Sci., 56, 711-723, 1999.

Zhang, D.-L., Tian, L., and Yang, M.-J.: Genesis of Typhoon Nari (2001) from a mesoscale convective system, J. Geophys. Res., 116, D23104, doi:10.1029/2011JD016640, 2011.

Zipser, E. J.: Mesoscale and convective-scale downdrafts as distinct components of squall-line structure, Mon. Weather Rev., 105, 1568-1589, 1977. 